\title{
Reduced modular symmetries of threshold corrections and gauge coupling unification
}

\author{
David Bailin and Alex Love \\ Department of Physics $\&$ Astronomy, University of Sussex, \\ Brighton, BN1 9QH U.K. \\ E-mail: d.bailin@sussex.ac.uk
}

ABSTRACT: We revisit the question of gauge coupling unification at the string scale in orbifold compactifications of the heterotic string for the supersymmetric Standard Model. In the presence of discrete Wilson lines threshold corrections with modular symmetry that is a subgroup of the full modular group arise. We find that reduced modular symmetries not previously reported are possible. We conjecture that the effects of such threshold corrections can be simulated using sums of terms built from Dedekind eta functions to obtain the appropriate modular symmetry. For the cases of the $\mathbb{Z}_{8}$-I orbifold and the $\mathbb{Z}_{3} \times \mathbb{Z}_{6}$ orbifold it is easily possible to obtain gauge coupling unification at the "observed" scale with Kähler moduli $T$ of approximately one.

KEYWORDS: Strings and branes phenomenology, Supersymmetry Phenomenology

ARXIV EPRINT: 1412.7327 


\section{Contents}

1 Introduction 1

2 Duality symmetry and Wilson lines 4

$2.1 \mathbb{Z}_{6}$-planes 6

$\begin{array}{lll}2.2 & \mathbb{Z}_{4} \text {-planes } & 7\end{array}$

$2.3 \mathbb{Z}_{3}$-planes 8

3 Gauge coupling constant unification $\quad 8$

$\begin{array}{lr}3.1 \text { The } \mathbb{Z}_{8} \text {-I orbifold } & 10\end{array}$

$\begin{array}{lll}3.2 & \text { The } \mathbb{Z}_{3} \times \mathbb{Z}_{6} \text { orbifold } & 13\end{array}$

4 Estimation of the Kähler modulus needed for unification 19

4.1 Unification in the $\mathbb{Z}_{8}$-I orbifold 20

4.2 Unification in the $\mathbb{Z}_{3} \times \mathbb{Z}_{6}$ orbifold 20

5 Conclusions $\quad 22$

\section{Introduction}

It is well known that when the $\mathrm{SU}(3)_{\text {colour }} \times \mathrm{SU}(2)_{L} \times \mathrm{U}(1)_{Y}$ gauge coupling constants $g_{1,2,3}$ are extrapolated to high energies using the renormalisation group equations of the supersymmetric Standard Model with just two Higgs doublets $[1,2]$ the three gauge couplings reach a common value $g\left(m_{X}\right)=g_{\text {string }} \simeq 0.7$ at a scale $m_{X} \simeq 2 \times 10^{16} \mathrm{GeV}$. (Arguably, this is the best evidence to date for the presence of supersymmetry underlying the Standard Model.) In contrast, in the context of heterotic string theory, unification is expected [3] at a scale $m_{\text {string }} \simeq 5.27 \times 10^{17} g_{\text {string }} \mathrm{GeV}$, a factor of order twenty or so larger than the "observed" unification scale. (Arguably, this is the best evidence to date that string theory is wrong!) However, the renormalisation group equations used in the extrapolation do not include contributions from the massive states associated with the unification threshold. In the case of string unification, since infinite towers of massive states contribute above the string threshold, one might hope for substantial contributions $\Delta_{a}$ from such loops to the renormalisation group equations for the gauge coupling constants $g_{a}(\mu),(a=1,2,3)$ of the (simple) group $G_{a}$

$$
\frac{1}{g_{a}^{2}(\mu)}=\frac{k_{a}}{g_{\text {string }}^{2}}+\frac{b_{a}}{16 \pi^{2}} \ln \frac{m_{\text {string }}^{2}}{\mu^{2}}+\frac{1}{16 \pi^{2}} \Delta_{a}
$$

Here $k_{a}$ is the affine level of the gauge group factor $G_{a}$,

$$
b_{a}=-3 C\left(G_{a}\right)+\sum_{\mathbf{R}_{a}} h_{\mathbf{R}_{a}} T\left(\mathbf{R}_{a}\right)
$$


is the one-loop beta function coefficient for $G_{a}$, with $C\left(G_{a}\right)$ the quadratic Casimir for the adjoint representation, $T\left(\mathbf{R}_{a}\right)=\operatorname{Tr} Q_{a}^{2}$ where $Q_{a}$ is any generator of $G_{a}$ in the representation $\mathbf{R}_{a}$, and $h_{\mathbf{R}_{a}}$ is the number of chiral multiplets in the representation $R_{a}$. We use the standard normalisation of the generators such that for the fundamental representation $\mathbf{N}$ of the group $\mathrm{SU}(N), T(\mathbf{N})=1 / 2$.

The threshold corrections $\Delta_{a}$ in general depend upon the various Kähler and complex structure moduli $T_{i}$ and $U_{j}$ associated with the 6-dimensional space on which the heterotic string is compactified, and it is conceivable that these corrections can bridge the gap between the observed unification scale $m_{X}$ and the string scale $m_{\text {string, }}$, at least for certain, hopefully realistic, values of the moduli. Of course, this is not the only approach to solving this problem. As discussed in [4], other possibilities include string GUT models, nonstandard affine levels, light supersymmetry thresholds, extra non-supersymmetric matter, no-supersymmetric strings, and strong-coupling effects in string theory.

The toroidal world sheet associated with a (closed) string loop is characterised by a modular parameter $\tau$ and invariance under $\operatorname{PSL}(2, \mathbb{Z})$ global modular transformations of the form

$$
\tau \rightarrow \frac{\alpha \tau-i \beta}{i \gamma \tau+\delta}
$$

with $\alpha, \beta, \gamma, \delta \in \mathbb{Z}$ and $\alpha \gamma-\beta \delta=1$ in turn induces target-space invariance under transformations of the moduli $T_{k}$ and $U_{k}$. Generically, this modular symmetry is also $\operatorname{PSL}(2, \mathbb{Z})$

$$
T_{k} \rightarrow \frac{a_{k} T_{k}-i b_{k}}{i c_{k} T_{k}+d_{k}}
$$

with $a_{k}, b_{k}, c_{k}, d_{k} \in \mathbb{Z}$ and $a_{k} d_{k}-b_{k} c_{k}=1$. Up to constant terms, independent of the moduli, the threshold correction arising from such an $\mathcal{N}=2$ sector has the form

$$
\Delta_{a}=-\left(b_{a}^{\prime k}-\delta_{\mathrm{GS}}^{k}\right) \ln \left[\left(T_{k}+\bar{T}_{k}\right)\left|\eta\left(T_{k}\right)\right|^{4}\right]
$$

with a similar contribution from the complex-structure modulus $U_{k}$. Here $\delta_{\mathrm{GS}}^{k}$ is the (universal) coefficient of the Green-Schwarz term, and $\eta(T)$ is the Dedekind eta function

$$
\eta(T):=e^{-\pi T / 12} \prod_{n=1}^{\infty}\left(1-e^{-2 \pi n T}\right) .
$$

Note that for large values of $T$ the eta function $\eta(T) \sim e^{-\pi T / 12}$, so that $\Delta_{a} \propto T$. It is this feature that is exploited in attempts to bridge the gap between $m_{X}$ and $m_{\text {string. Under a }}$ modular transformation of the form given in eq. (1.4)

$$
\begin{aligned}
T+\bar{T} & \rightarrow \frac{T+\bar{T}}{|i c T+d|^{2}} \\
\eta(T) & \rightarrow(i c T+d)^{1 / 2} \eta(T)
\end{aligned}
$$

up to a constant, so that $\Delta_{a}$ is indeed invariant.

In this paper we are concerned only with orbifold compactifications in which the 6torus $T^{6}$ is quotiented with a $\mathbb{Z}_{N}$ or $\mathbb{Z}_{N} \times \mathbb{Z}_{M}$ point group. For most of these there are 
$\theta^{p}$-twisted sectors of the generator(s) $\theta$ of the point group that leave a single plane invariant; such twisted sectors are referred to as $\mathcal{N}=2$ sectors. The calculation of the contribution of a twisted sector with an invariant plane $T_{k}^{2}$ to the threshold corrections entails a sum over the momentum $\left(m_{2 k-1}, m_{2 k}\right)$ and winding numbers $\left(n_{2 k-1}, n_{2 k}\right)$ that arise in the zeromode expansion of the string world sheet in such sectors, and it is this that generates the dependence on the moduli $T_{k}$ and $U_{k}$. The foregoing result applies whenever the $\mathcal{N}=2$ sector fixed planes are such that the torus $T^{6}$ on which the point group action is realised can be decomposed into a direct product $T^{4} \oplus T^{2}$ with the fixed plane lying in $T^{2}$. This is certainly not the case for all orbifolds. Consider, for example, the $\mathbb{Z}_{6}$-II orbifold. It has point-group generator $\theta$ with eigenvalues $e^{2 \pi i v_{k}},(k=1,2,3)$ where

$$
\left(v_{1}, v_{2}, v_{3}\right)=\frac{1}{6}(2,1,-3) .
$$

The symmetry may be realised on several lattices, but in particular on the $\mathrm{SU}(3) \oplus G_{2} \oplus$ $\mathrm{SO}(4)$ and $\mathrm{SU}(6) \oplus \mathrm{SU}(2)$ lattices; in both cases the action of the point-group is given by the Casimir operators of the various groups. Evidently the $\theta^{2}, \theta^{4}$ and $\theta^{3}$-twisted sectors all have fixed planes. In the first realisation, the lattice is the product of three 2-tori $T_{1}^{2} \oplus T_{2}^{2} \oplus T_{3}^{2}$, with $T_{3}^{2}$ the fixed plane of $\theta^{2}, \theta^{4}$, and $T_{1}^{2}$ the fixed plane of $\theta^{3}$. Since $\theta$ acts as $\mathbb{Z}_{3}$ on $T_{1}^{2}$, the complex structure $U_{1}=e^{2 \pi i / 3}$ is fixed and there is no associated modular symmetry. On $T_{3}^{2}$, however, $\theta$ acts as a reflection $\mathbb{Z}_{2}$ and the complex structure $U_{3}$ is arbitrary. In the first case, the above analysis applies, and there is the full $\operatorname{PSL}(2, \mathbb{Z})$ modular symmetry for the Kähler moduli $T_{1}$ and $T_{3}$, as well as for the complex structure $U_{3}$. (Of course, there is also a Kähler modulus $T_{2}$ associated with the second plane, but since this is not an $\mathcal{N}=2$ plane, it does not enter the threshold corrections.) However, in the second case the lattice is $T^{5} \oplus T^{1}$ and the previous analysis does not apply. Such cases must be treated on a case by case basis, and in this case it was shown [5] that the modular group associated with $T_{1}$ is $\Gamma^{0}(2)$, meaning the subgroup of $\operatorname{PSL}(2, \mathbb{Z})$ defined in eq. (1.4) with the integer $b_{1}=0 \bmod 2$. Similarly, the modular group associated with $T_{3}$ is $\Gamma^{0}(3)$, corresponding to the subgroup with $b_{3}=0 \bmod 3$. The modular symmetry associated with $U_{3}$ was shown to be $\Gamma_{0}(3)$, corresponding to $c_{3}^{\prime}=0 \bmod 3$, with $c_{3}^{\prime}$ the analogue for $U_{3}$ of $c_{3}$. As in the separable case, the threshold corrections $\Delta_{a}$ may be calculated and are indeed invariant under the reduced modular symmetries. For example, the $T_{3}$ - and $U_{3}$-dependent threshold corrections are [6]

$$
\begin{aligned}
\Delta_{a}= & -2 b_{a}^{\left(1, \theta^{2}\right)} \ln \left[\left(T_{3}+\bar{T}_{3}\right)\left|\eta\left(T_{3}\right)\right|^{4}\left(U_{3}+\bar{U}_{3}\right)\left|\eta\left(U_{3}\right)\right|^{4}\right] \\
& -2 b_{a}^{\left(1, \theta^{2}\right)} \ln \left[\left(T_{3}+\bar{T}_{3}\right)\left|\eta\left(\frac{T_{3}}{3}\right)\right|^{4}\left(U_{3}+\bar{U}_{3}\right)\left|\eta\left(3 U_{3}\right)\right|^{4}\right] .
\end{aligned}
$$

The PSL $(2, \mathbb{Z})$ modular symmetries are also broken by the presence of discrete background Wilson lines [7-11]. Besides the standard degrees of freedom $X^{i}, \Psi^{i}$ associated with the 10-dimensional string, the heterotic string has 16 additional left-moving bosonic degrees of freedom $X^{I}(\tau+\sigma)$ on the $E_{8} \times E_{8}$ lattice. Background Wilson lines $A_{a}^{I}$ couple the $X^{I}$ to the six bosonic degrees of freedom $X^{a}$ associated with the (orbifold) compactified 
dimensions via a term in the world-sheet action

$$
S \supset \frac{1}{2 \pi} \int d \tau d \sigma \epsilon^{\alpha \beta} A_{a}^{I} \partial_{\alpha} X^{a} \partial_{\beta} X^{I} .
$$

This has the effect of shifting the canonical left- and right-moving momenta by terms involving the quantised momentum on the $E_{8} \times E_{8}$ lattice, but also of shifting the lattice momentum by terms involving the winding numbers. It is this mixing that modifies the modular-invariance properties. However, unlike the situation in the absence of Wilson lines, to our knowledge the string loop threshold corrections in the presence of discrete background Wilson lines have not actually been calculated. ${ }^{1}$ A systematic study of the modular symmetries in the presence of discrete Wilson lines was made in [13] for all Abelian orbifolds whose point-group action is realised by Coxeter elements or their generalisations.

In this paper we shall revisit this question, but only for those orbifolds in which the $\mathcal{N}=2$ fixed planes are separable into the $T^{2}$ part of the 6 -torus $T^{4} \oplus T^{2}$ on which the point group is realised. We also restrict ourselves to models with $n o \mathbb{Z}_{2}$ fixed planes; as is apparent from the previous treatment [13], such models are considerably more complicated. In the notation of that paper, only (certain compactifications of) the $\mathbb{Z}_{6}$-I, $\mathbb{Z}_{8}$-I, $\mathbb{Z}_{12}$-I, $\mathbb{Z}_{3} \times \mathbb{Z}_{3}$, $\mathbb{Z}_{4} \times \mathbb{Z}_{4}, \mathbb{Z}_{3} \times \mathbb{Z}_{6}$ and $\mathbb{Z}_{6} \times \mathbb{Z}_{6}$ orbifolds satisfy these criteria. We also speculate on the effect that our new results might have on the question of whether the threshold corrections can bridge the gap between the observed unification scale $m_{X}$ of the supersymmetric Standard Model and $m_{\text {string. }}$. In the absence of discrete Wilson lines, such unification can only be achieved with large values $T \sim 26$ of the Kähler moduli [9]. In any case, it was also shown that this unification is not possible for the $\mathbb{Z}_{6}$-I or $\mathbb{Z}_{12}$-I orbifolds. Further, for the $\mathbb{Z}_{N} \times \mathbb{Z}_{M}$ orbifolds, whose threshold corrections depend upon all three Kähler moduli $T_{1,2,3}$, it is reasonable, in our view, to insist that unification is achievable with all three moduli of comparable size $T_{1} \simeq T_{2} \simeq T_{3}$, in which case of these only the $\mathbb{Z}_{3} \times \mathbb{Z}_{6}$ survives. Of course, one may take the opposite view to ours and make a virtue of highly anisotropic compactifications; see, for example, Hebecker \& Trapletti [14].

Thus, in the end, we pursue the question of threshold corrections just for the $\mathbb{Z}_{8}$-I and $\mathbb{Z}_{3} \times \mathbb{Z}_{6}$ orbifolds. We shall argue that the considerably reduced modular symmetry that we now find can arise when discrete Wilson lines are present allows unification in both models with Kähler moduli $T \sim 1$ of order unity, if we conjecture that the effect of such threshold corrections can be simulated using sums of terms built from Dedekind eta functions. There have been some attempts to construct realistic models on both of these orbifolds; see, for example, Groot Nibbelink \& Loukas [15] on the $\mathbb{Z}_{8}$ orbifold and Nilles \& Vaudrevange [16] on the $\mathbb{Z}_{N} \times \mathbb{Z}_{M}$ orbifolds, and it will be interesting to see whether our results apply to their models.

\section{Duality symmetry and Wilson lines}

The action of the point group generator $\theta$ of a $\mathbb{Z}_{N}$ orbifold on the defining lattice basis $\mathbf{e}_{a},(a=1,2, \ldots, 6)$, is given by the elements of a matrix $Q$ defined by

$$
\theta \mathbf{e}_{a}=Q_{b a} \mathbf{e}_{b} .
$$

\footnotetext{
${ }^{1}$ The matter was addressed by Klaput \& Paleani in 2010 [12], but we are unclear as to the status of this paper since it has not been published. We are grateful to the referee for drawing our atention to it.
} 
The $16 \times 6$ dimensional Wilson line matrix $A$ with elements $A_{a}^{I}$ may be written as

$$
A:=\left(\mathbf{A}_{1}, \mathbf{A}_{2}, \mathbf{A}_{3}, \mathbf{A}_{4}, \mathbf{A}_{5}, \mathbf{A}_{6}\right)
$$

where $\mathbf{A}_{a}$ are 16-dimensional column vectors. It is required to satisfy [10]

$$
A(1-Q) \in \mathbb{Z}
$$

and this leads to the constraints on the vectors $\mathbf{A}_{a}$ that are listed in appendix B of [13]. $A$ must also satisfy

$$
A^{t} C A(1-Q)+\left(1-Q^{*}\right) A^{t} C A \in 2 \mathbb{Z}
$$

where $C$ is the Cartan matrix for $E_{8} \times E_{8}$ and

$$
Q^{*}:=\left(Q^{t}\right)^{-1}
$$

For the $\mathbb{Z}_{N} \times \mathbb{Z}_{M}$ orbifolds, analogous constraints apply to the matrices $Q_{\theta}$ and $Q_{\omega}$ representing the two generators $\theta$ and $\omega$.

As detailed in appendix B of [13], the fixed planes of the various orbifolds may be classified according to how the point-group generator(s) acts on that plane. The only possibilities are $\mathbb{Z}_{2}, \mathbb{Z}_{3}, \mathbb{Z}_{4}$ and $\mathbb{Z}_{6}$. How they arise in the $T^{4} \oplus T^{2}$ separable orbifolds is indicated in table 1. Also displayed are the restrictions following from eq. (2.3) on the Wilson lines $\left(\mathbf{A}_{2 k-1}, \mathbf{A}_{2 k}\right)$ in that plane. (The twists associated with the point-group generators $\theta$ (and $\omega$ ) are listed in appendix A of [13], for example.)

The modular symmetry of the the Kähler moduli $T_{k}$ associated with the fixed torus $T_{k}^{2}$, in which $T_{k}$ transforms as in eq. (1.4), is constrained [11] by

$$
\begin{array}{r}
c_{k}\left(\mathbf{A}_{2 k-1}, \mathbf{A}_{2 k}\right) \in \mathbb{Z} \\
c_{k}\left(\begin{array}{c}
\left.\mathbf{A}_{2 k-1}^{t}\right) \\
\mathbf{A}_{2 k}^{t}
\end{array}\right) C\left(\mathbf{A}_{2 k-1}, \mathbf{A}_{2 k}\right) \in 2 \mathbb{Z} \\
c_{k}\left(\mathbf{A}_{2 k-1}, \mathbf{A}_{2 k}\right) J\left(\begin{array}{c}
\left.\mathbf{A}_{2 k-1}^{t}\right) \\
\mathbf{A}_{2 k}^{t}
\end{array}\right) C \in \mathbb{Z} \\
2\left(1-d_{k}\right)\left(\mathbf{A}_{2 k-1}, \mathbf{A}_{2 k}\right)-c_{k}\left(\mathbf{A}_{2 k-1}, \mathbf{A}_{2 k}\right) J\left(\begin{array}{c}
\mathbf{A}_{2 k-1}^{t} \\
\mathbf{A}_{2 k}^{t}
\end{array}\right) C\left(\mathbf{A}_{2 k-1}, \mathbf{A}_{2 k}\right) \in 2 \mathbb{Z} \\
2\left(1-a_{k}\right) C\left(\mathbf{A}_{2 k-1}, \mathbf{A}_{2 k}\right)-c_{k} C\left(\mathbf{A}_{2 k-1}, \mathbf{A}_{2 k}\right) J\left(\begin{array}{c}
\mathbf{A}_{2 k-1}^{t} \\
\mathbf{A}_{2 k}^{t}
\end{array}\right) C\left(\mathbf{A}_{2 k-1}, \mathbf{A}_{2 k}\right) \in 2 \mathbb{Z} \\
2\left(2-a_{k}-d_{k}\right)\left(\begin{array}{c}
\mathbf{A}_{2 k-1}^{t} \\
\mathbf{A}_{2 k}^{t}
\end{array}\right) C\left(\mathbf{A}_{2 k-1}, \mathbf{A}_{2 k}\right) \\
-c_{k}\left(\begin{array}{c}
\mathbf{A}_{2 k-1}^{t} \\
\mathbf{A}_{2 k}^{t}
\end{array}\right) C\left(\mathbf{A}_{2 k-1}, \mathbf{A}_{2 k}\right) J\left(\begin{array}{c}
\mathbf{A}_{2 k-1}^{t} \\
\mathbf{A}_{2 k}^{t}
\end{array}\right) C\left(\mathbf{A}_{2 k-1}, \mathbf{A}_{2 k}\right) \in 4 \mathbb{Z}
\end{array}
$$




\begin{tabular}{|c|c|c|c|}
\hline $\mathbb{Z}_{N}$ fixed plane & Orbifold & Twisted sector & $\left(\mathbf{A}_{2 k-1}, \mathbf{A}_{2 k}\right)$ \\
\hline $\mathbb{Z}_{2}$ & $\mathbb{Z}_{4}$ & $\theta^{2}$ & $2 \mathbf{A}_{3}=0 \bmod 1=2 \mathbf{A}_{6}$ \\
& $\mathbb{Z}_{6}$-II & $\theta^{2}$ & $2 \mathbf{A}_{5}=0 \bmod 1=2 \mathbf{A}_{6}$ \\
& $\mathbb{Z}_{8}$-II & $\theta^{2}$ & $2 \mathbf{A}_{5}=0 \bmod 1=2 \mathbf{A}_{6}$ \\
& $\mathbb{Z}_{12}$-II & $\theta^{2}$ & $2 \mathbf{A}_{5}=0 \bmod 1=2 \mathbf{A}_{6}$ \\
\hline $\mathbb{Z}_{3}$ & $\mathbb{Z}_{6}$-I,II & $\theta^{3}$ & $\mathbf{A}_{1}-\mathbf{A}_{2}=0 \bmod 1,3 \mathbf{A}_{1}=0 \bmod 1$ \\
& $\mathbb{Z}_{12}$-I & $\theta^{3}$ & $\mathbf{A}_{5}-\mathbf{A}_{6}=0 \bmod 1,3 \mathbf{A}_{5}=0 \bmod 1$ \\
& $\mathbb{Z}_{3} \times \mathbb{Z}_{3}$ & $\omega^{k}$ & $\mathbf{A}_{1}-\mathbf{A}_{2}=0 \bmod 1,3 \mathbf{A}_{1}=0 \bmod 1$ \\
& & $\theta^{k}$ & $\mathbf{A}_{3}-\mathbf{A}_{4}=0 \bmod 1,3 \mathbf{A}_{3}=0 \bmod 1$ \\
& & $\left(\theta \omega^{2}\right)^{k}$ & $\mathbf{A}_{5}-\mathbf{A}_{6}=0 \bmod 1,3 \mathbf{A}_{5}=0 \bmod 1$ \\
& $\mathbb{Z}_{3} \times \mathbb{Z}_{6}$ & $\omega^{k}$ & $\mathbf{A}_{1}-\mathbf{A}_{2}=0 \bmod 1,3 \mathbf{A}_{1}=0 \bmod 1$ \\
\hline $\mathbb{Z}_{4}$ & $\mathbb{Z}_{8}$ I & $\mathbf{A}_{5}=0 \bmod 1=2 \mathbf{A}_{6}$ \\
& $\mathbb{Z}_{4} \times \mathbb{Z}_{4}$ & $\omega^{k}$ & $\mathbf{A}_{1}=0 \bmod 1=2 \mathbf{A}_{2}$ \\
& & $\theta^{k}$ & $\mathbf{A}_{3}=0 \bmod 1=2 \mathbf{A}_{4}$ \\
& & $\left(\theta^{2} \omega^{2}\right)^{k}$ & $\mathbf{A}_{5}=0 \bmod 1=2 \mathbf{A}_{6}$ \\
\hline $\mathbb{Z}_{6}$ & $\mathbb{Z}_{3} \times \mathbb{Z}_{6}$ & $\theta^{k}$ & $\mathbf{A}_{3}=0 \bmod 1=\mathbf{A}_{4}$ \\
& & $\left(\theta \omega^{4}\right)^{k}$ & $\mathbf{A}_{5}=0 \bmod 1=\mathbf{A}_{6}$ \\
& $\mathbb{Z}_{6} \times \mathbb{Z}_{6}$ & $\omega^{k}$ & $\mathbf{A}_{1}=0 \bmod 1=\mathbf{A}_{2}$ \\
& & $\theta^{k}$ & $\mathbf{A}_{3}=0 \bmod 1=\mathbf{A}_{4}$ \\
& & $\left(\theta \omega^{5}\right)^{k}$ & $\mathbf{A}_{5}=0 \bmod 1=\mathbf{A}_{6}$ \\
\hline
\end{tabular}

Table 1. Classification of fixed planes by the action of the point group on that plane.

where

$$
J:=\left(\begin{array}{cc}
0 & 1 \\
-1 & 0
\end{array}\right)
$$

We shall discuss in turn the modular symmetries of the three non- $\mathbb{Z}_{2}$ fixed plane classes displayed in table 1.

\section{$2.1 \quad \mathbb{Z}_{6}$-planes}

Consider first the $\mathbb{Z}_{6}$-plane $T_{2}^{2}$ arising in the $\theta^{k}$-twisted sectors of the $\mathbb{Z}_{3} \times \mathbb{Z}_{6}$ orbifold. Eq. (2.3) requires only that the Wilson lines in that torus are $E_{8} \times E_{8}$ lattice vectors. They are also constrained by eq. (2.4) which in this case gives

$$
\left(\begin{array}{cc}
\mathbf{A}_{3}^{t} C \mathbf{A}_{3} & -\mathbf{A}_{3}^{t} C \mathbf{A}_{3}-\mathbf{A}_{4}^{t} C \mathbf{A}_{4} \\
\mathbf{A}_{3}^{t} C \mathbf{A}_{3}+2 \mathbf{A}_{3}^{t} C \mathbf{A}_{4}+\mathbf{A}_{4}^{t} C \mathbf{A}_{4} & \mathbf{A}_{4}^{t} C \mathbf{A}_{4}
\end{array}\right) \in 2 \mathbb{Z}
$$

which is automatically satisfied when $\mathbf{A}_{3}$ and $\mathbf{A}_{4}$ are lattice vectors. Eq. (2.6) is also satisfied since $c_{2}$ is an integer. However, a new constraint arises from eq. (2.7), namely that

$$
c_{2} \mathbf{A}_{3}^{t} C \mathbf{A}_{4} \in 2 \mathbb{Z}
$$

Since $\mathbf{A}_{3}$ and $\mathbf{A}_{4}$ are lattice vectors, $\mathbf{A}_{3}^{t} C \mathbf{A}_{4} \in \mathbb{Z}$, so if $\mathbf{A}_{3}^{t} C \mathbf{A}_{4}$ is even, then eq. (2.8) is satisfied without any further constraint on $c_{2}$. Alternatively, if $\mathbf{A}_{3}^{t} C \mathbf{A}_{4}$ is odd, then 
we require that $c_{2}=0 \bmod 2$. In both cases, eqs. (2.8)-(2.10) are then satisfied without further constraint. If $\mathbf{A}_{3}^{t} C \mathbf{A}_{4}$ is even, eq. (2.11) is also satisfied without further constraint. When $\mathbf{A}_{3}^{t} C \mathbf{A}_{4}$ is odd, then since $c_{2}$ must be even it follows from the unimodularity that $a_{2}$ and $d_{2}$ are both odd, and this ensures that the first term on the left-hand side of eq. (2.11) is $0 \bmod 4$. However, ensuring that the second term is also $0 \bmod 4$ requires that $c_{2}=0 \bmod 4$. Thus there are two generic cases for the modular symmetry of $T_{2}$. If $\mathbf{A}_{3}^{t} C \mathbf{A}_{4}$ is even, then it is the full $\operatorname{PSL}(2, \mathbb{Z})$. Otherwise it is $\Gamma_{0}(4)$, the subgroup of $\operatorname{PSL}(2, \mathbb{Z})$ with $c_{2}=0 \bmod 4$. This alternative was overlooked in reference [13].

The torus $T_{3}^{2}$ is fixed in the $\left(\theta \omega^{4}\right)^{k}$-twisted sectors. Since the action of $\theta$ in $T_{3}^{2}$ is just the square of the action of $\omega$, this too is a $\mathbb{Z}_{6}$-plane. The action of $\omega$ in $T_{3}^{2}$ is the same as its action in $T_{2}^{2}$, so the constraint deriving from eq. (2.4) is the same as that displayed in eq. (2.13) but with $\left(\mathbf{A}_{5}, \mathbf{A}_{6}\right)$ replacing $\left(\mathbf{A}_{3}, \mathbf{A}_{4}\right)$. It follows again that there are two generic cases for the modular symmetry of $T_{3}$. If $\mathbf{A}_{5}^{t} C \mathbf{A}_{6}$ is even, then it is the full $\operatorname{PSL}(2, \mathbb{Z})$. Otherwise it is $\Gamma_{0}(4)$. The same analysis applies to the three $\mathbb{Z}_{6}$-planes of the $\mathbb{Z}_{6} \times \mathbb{Z}_{6}$ orbifold, so the conclusion is that these are the two generic symmetries for all (Kähler) moduli in $\mathbb{Z}_{6}$-planes.

\section{$2.2 \quad \mathbb{Z}_{4}$-planes}

For the fixed plane $T_{3}^{2}$ that arises in the $\theta^{4}$-twisted sector of the $\mathbb{Z}_{8}$ orbifold, the first $\mathbb{Z}_{4}$-plane entry in table 1 , the constraint given in eq. (2.4) yields

$$
\left(\begin{array}{cc}
2 \mathbf{A}_{5}^{t} C \mathbf{A}_{5} & \mathbf{A}_{5}^{t} C \mathbf{A}_{5}+4 \mathbf{A}_{5}^{t} C \mathbf{A}_{6}+2 \mathbf{A}_{6}^{t} C \mathbf{A}_{6} \\
-\mathbf{A}_{5}^{t} C \mathbf{A}_{5}-2 \mathbf{A}_{6}^{t} C \mathbf{A}_{6} & 2 \mathbf{A}_{6}^{t} C \mathbf{A}_{6}
\end{array}\right) \in 2 \mathbb{Z}
$$

where $\mathbf{A}_{5}$ and $2 \mathbf{A}_{6}$ are lattice vectors. This is satisfied provided only that

$$
\mathbf{A}_{6}^{t} C \mathbf{A}_{6} \in \mathbb{Z} .
$$

If $\mathbf{A}_{6}$ is a lattice vector, then eq. (2.16) is automatically satisfied, and the analysis of the modular constraints is the same as that given above for the $\mathbb{Z}_{6}$-planes. In this case, then, the conclusions are identical.

We therefore need only consider the alternative, namely that $\mathbf{A}_{6}$ is not a lattice vector. In this case, eq. (2.6) requires that $c_{3}=0 \bmod 2$. Then, the only further constraint from eq. (2.7) is that $c_{3} \mathbf{A}_{5}^{t} C \mathbf{A}_{6}$ is even. Now, $\mathbf{A}_{5}^{t} C \mathbf{A}_{6}$ is either an integer or half-integer. If the former, then there is no further constraint on $c_{3}$. However, if $\mathbf{A}_{5}^{t} C \mathbf{A}_{6} \in \mathbb{Z}+\frac{1}{2}$, then we require that $c_{3}=0 \bmod 4$. In both cases, eq. (2.8) is satisfied without further constraint. Since $c_{3}$ is even, unimodularity requires that $a_{3}$ and $d_{3}$ are both odd, and the first term on the left-hand side of eqs. (2.9) and (2.10) are both even. Then, to satisfy eq. (2.9) we only require that $c_{3} \mathbf{A}_{5}^{t} C \mathbf{A}_{6}=0 \bmod 4$. If $\mathbf{A}_{5}^{t} C \mathbf{A}_{6}$ is even, this is already satisfied. However, if $\mathbf{A}_{5}^{t} C \mathbf{A}_{6}$ is odd, we now require that $c_{3}=0 \bmod 4$, whereas if $\mathbf{A}_{5}^{t} C \mathbf{A}_{6} \in \mathbb{Z}+\frac{1}{2}$, then we require that $c_{3}=0 \bmod 8$. These are sufficient to ensure that eq. (2.10) is fully satisfied and, except in the third eventuality, that eq. (2.11) is too. In the case that $\mathbf{A}_{5}^{t} C \mathbf{A}_{6} \in \mathbb{Z}+\frac{1}{2}$, this requires that $c_{3}=0 \bmod 16$. Thus, when $\mathbf{A}_{6}$ is not a lattice vector the conclusions are that (i) if $\mathbf{A}_{5}^{t} C \mathbf{A}_{6}$ is even, the modular group for $T_{3}$ is $\Gamma_{0}(2)$, (ii) if $\mathbf{A}_{5}^{t} C \mathbf{A}_{6}$ is odd, then the modular group is $\Gamma_{0}(4)$, but (iii) if $\mathbf{A}_{5}^{t} C \mathbf{A}_{6} \in \mathbb{Z}+\frac{1}{2}$, then it is $\Gamma_{0}(16)$. 


\section{$2.3 \quad \mathbb{Z}_{3}$-planes}

For the fixed plane $T_{1}^{2}$ that arises in the $\omega^{k}$-twisted sector of the $\mathbb{Z}_{3} \times \mathbb{Z}_{6}$ orbifold, the last $\mathbb{Z}_{3}$-plane entry in table 1 , the constraint given in eq. (2.4) yields

$$
\left(\begin{array}{cc}
3 \mathbf{A}_{1}^{t} C \mathbf{A}_{1} & \mathbf{A}_{1}^{t} C \mathbf{A}_{1}+4 \mathbf{A}_{1}^{t} C \mathbf{A}_{2}+\mathbf{A}_{2}^{t} C \mathbf{A}_{2} \\
2 \mathbf{A}_{1}^{t} C \mathbf{A}_{2}-\mathbf{A}_{1}^{t} C \mathbf{A}_{1}-\mathbf{A}_{2}^{t} C \mathbf{A}_{2} & 3 \mathbf{A}_{2}^{t} C \mathbf{A}_{2}
\end{array}\right) \in 2 \mathbb{Z}
$$

where $\mathbf{A}_{1}$ and $\mathbf{A}_{2}$ differ by a lattice vector and $3 \mathbf{A}_{1}$ is a lattice vector. As above, if $\mathbf{A}_{1}$, and hence $\mathbf{A}_{2}$, are lattice vectors, then this is automatically satisfied, and the analysis of the modular constraints is the same as for the $\mathbb{Z}_{4}$-planes. The conclusions, in this case for $T_{1}$, are the same.

We therefore need only consider the alternative, namely that $\mathbf{A}_{1}$ is not a lattice vector. Then to satisfy eq. (2.17) we must have that

$$
3 \mathbf{A}_{1}^{t} C \mathbf{A}_{1} \in 2 \mathbb{Z} .
$$

We may write

$$
\mathbf{A}_{2}-\mathbf{A}_{1}:=\mathbf{V}
$$

where $\mathbf{V} \in \mathbb{Z}$ is a lattice vector, so that $\mathbf{V}^{t} C \mathbf{V} \in 2 \mathbb{Z}$; it follows that $3 \mathbf{A}_{2}^{t} C \mathbf{A}_{2}$ is also even, since $3 \mathbf{A}_{1}^{t} C \mathbf{V}$ is an integer. Eq. (2.6) requires that $c_{1}=0 \bmod 3$. Then, the only further constraint from eq. (2.7) is that $c_{1} \mathbf{A}_{1}^{t} C \mathbf{V}=0 \bmod 2$. So, if $3 \mathbf{A}_{1}^{t} C \mathbf{V}=3 \mathbf{A}_{1}^{t} C \mathbf{A}_{2} \bmod 2$ is even, there is no further constraint on $c_{1}$, but if $3 \mathbf{A}_{1}^{t} C \mathbf{V}$ is odd, then we require that $c_{1}$ is even, and hence $c_{1}=0 \bmod 6$. As before, eq. (2.8) is then satisfied without further constraint. In this case, unimodularity now means that we may choose that $a_{1}=1 \bmod 3=$ $d_{1}$, which ensures that the first term on the left-hand sides of eqs. (2.9) and (2.10) are even. Then to satisfy them we require only that $c_{1} \mathbf{A}_{1}^{t} C \mathbf{V}=0 \bmod 6$. Thus, if $3 \mathbf{A}_{1}^{t} C \mathbf{V}=0 \bmod 6$ there is no further constraint; if $3 \mathbf{A}_{1}^{t} C \mathbf{V}=0 \bmod 2($ but not $0 \bmod 6)$, then $c_{1}=0 \bmod 9$. However if $3 \mathbf{A}_{1}^{t} C \mathbf{V}$ is odd, we require that $c_{1}=0 \bmod 18$. Finally, unimodularity requires that $2-a_{1}-d_{1}=0 \bmod c_{1}$, and to satisfy eq. (2.11) we require only that $c_{1}\left(\mathbf{A}_{1}^{t} C \mathbf{V}\right)^{2}=$ $0 \bmod 4$. In the case that $3 \mathbf{A}_{1}^{t} C \mathbf{V}$ is even, there is no further constraint on $c_{1}=0 \bmod 9$. However, if $3 \mathbf{A}_{1}^{t} C \mathbf{V}$ is odd, we now require that $c_{1}=0 \bmod 36$. In the former case, the modular symmetry for $T_{1}$ is $\Gamma_{0}(9)$, and in the latter it is $\Gamma_{0}(36)$.

It is the availability of these large integers $n$ in the reduced modular symmetry $\Gamma_{0}(n)$ of the $\mathbb{Z}_{8}$ and $\mathbb{Z}_{3} \times \mathbb{Z}_{6}$ orbifolds that we believe may enable the gap between $m_{X}$ and $m_{\text {string }}$ to be bridged.

\section{Gauge coupling constant unification}

The question we shall now address is whether the inclusion of the moduli-dependent threshold corrections in the renormalisation group equations (1.1) allows unification of the Standard Model gauge coupling constants $g_{a}(\mu),(a=1,2,3)$ at a scale $m_{X} \simeq 2 \times 10^{16} \mathrm{GeV}$ consistently with the string scale $m_{\text {string }} \simeq 3.7 \times 10^{17} \mathrm{GeV}$. Taking all groups at level 1 , this requires that for $a, b=1,2,3$

$$
\frac{m_{\text {string }}^{2}}{m_{X}^{2}}=\exp \left(-\frac{\Delta_{a}-\Delta_{b}}{b_{a}-b_{b}}\right)
$$


and consistency requires that

$$
\frac{\Delta_{3}-\Delta_{2}}{\Delta_{3}-\Delta_{1}}=\frac{b_{3}-b_{2}}{b_{3}-b_{1}}=\frac{5}{12}
$$

in the Standard Model since

$$
b_{3}=-3, \quad b_{2}=1, \quad b_{1}=\frac{33}{5} .
$$

Since $m_{\text {string }}>m_{X}$, we also require that

$$
\Delta_{3}-\Delta_{2}>0
$$

so that the exponent in eq. (3.1) is positive.

As we have already noted, the precise form of the threshold corrections in the presence of discrete Wilson lines is not known. ${ }^{2}$ However, since we know their modular symmetry, we may make plausible hypotheses of their form. In this paper we are only concerned with orbifolds in which there are no $\mathbb{Z}_{2}$ planes, in which case the complex structure moduli are fixed and only the modular symmetry of the Kähler moduli is relevant. In analogy with eq. (1.10) we assume ${ }^{3}$ that $\Delta_{a}$ has the general form

$$
\Delta_{a}=-\sum_{k=1}^{3}\left(b_{a}^{\prime k}-\delta_{\mathrm{GS}}^{k}\right) \ln \left[\left(T_{k}+\bar{T}_{k}\right)\left|\eta\left(p_{k} T_{k}\right) \eta\left(q_{k} T_{k}\right)\right|^{2}\right]
$$

where $p_{k}$ and $q_{k}$ are integers consistent with the modular symmetry in the $\mathcal{N}=2$ plane $T_{k}^{2}$. The coefficients $b_{a}^{\prime k}$ are given by the general formula [9]

$$
b_{a}^{\prime k}=-c\left(G_{a}\right)+\sum_{\mathbf{R}_{a}} T\left(\mathbf{R}_{a}\right)\left(1+2 n_{\mathbf{R}_{a}}^{k}\right) .
$$

Thus,

$$
\begin{aligned}
& {b_{3}^{\prime}}^{k}=3+\sum_{g=1}^{3}\left(2 n_{Q_{g}}^{k}+n_{u_{g}}^{k}+n_{d_{g}}^{k}\right) \\
& {b_{2}^{\prime}}^{k}=5+n_{h}^{k}+n_{h}^{k}+\sum_{g=1}^{3}\left(3 n_{Q_{g}}^{k}+n_{L_{g}}^{k}\right) \\
& {b_{1}^{\prime}}^{k}=\frac{33}{5}+\frac{3}{5}\left(n_{h}^{k}+n_{h}^{k}\right)+\frac{1}{5} \sum_{g=1}^{3}\left(n_{Q_{g}}^{k}+8 n_{u_{g}}^{k}+2 n_{d_{g}}^{k}+3 n_{L_{g}}^{k}+6 n_{\ell_{g}}^{k}\right)
\end{aligned}
$$

where $g=1,2,3$ labels the three Standard Model fermion generations, with $Q_{g}$ and $L_{g}$ respectively the quark and lepton doublets, $u_{g}, d_{g}$ and $\ell_{g}$ the quark and lepton singlets, and $h, \bar{h}$ are the two Higgs doublets; $n_{X}^{k}$ is the modular weight of the state $X$ under the modular transformation given in eq. (1.4).

\footnotetext{
${ }^{2}$ There has been recent progress in the evaluation of the one-loop modular integrals that are needed to determine the threshold corrections [17-20].

${ }^{3}$ The form assumed appears to be consistent with the results of [12].
} 


\subsection{The $\mathbb{Z}_{8}$-I orbifold}

The point group generator $\theta$ has eigenvalues $e^{2 \pi i v_{k}}$ with

$$
\left(v_{1}, v_{2}, v_{3}\right)=\frac{1}{8}(1,-3,2) .
$$

This may be realised by Casimirs acting on the $\mathrm{SO}(9) \times \mathrm{SO}(5)$ lattice or on the $\mathrm{SO}(8)^{[2]} \times$ $\mathrm{SO}(5)$ lattice, ${ }^{4}$ both of which have the form $T^{4} \times T^{2}$. In both there is a single fixed $\mathcal{N}=2$ plane $T_{3}^{2}$ in the $\theta^{4}$-twisted sector. Since $\theta$ acts as $\mathbb{Z}_{4}$ on this plane, only the Kähler modulus $T_{3}$ enters the threshold corrections. In this case eqs. (3.4) and (3.2) require that

$$
\begin{aligned}
& b_{3}^{\prime 3}-b_{2}^{\prime 3}>0 \\
& b_{3}^{\prime 3}-b_{1}^{\prime 3}>0 \\
& \frac{{b_{3}^{\prime}}^{3}-b_{2}^{\prime 3}}{{b_{3}^{\prime}}^{3}-b_{1}^{\prime 3}}=\frac{5}{12}
\end{aligned}
$$

and eqs. (3.7)-(3.9) give

$$
\begin{aligned}
{b_{3}^{\prime}}^{3}-b_{2}^{\prime 3} & =-2-Y^{3}+\sum_{g=1}^{3}\left(-n_{Q_{g}}^{3}+n_{u_{g}}^{3}\right)>0 \\
\frac{5}{3}\left(b_{3}^{\prime 3}-b_{1}^{\prime 3}\right) & =-6-Y^{3}+\sum_{g=1}^{3}\left(3 n_{Q_{g}}^{3}-n_{u_{g}}^{3}-2 n_{\ell_{g}}^{3}\right)>0
\end{aligned}
$$

where

$$
Y^{k}:=n_{h}^{k}+n_{\bar{h}}^{k}-\sum_{g=1}^{3}\left(n_{d_{g}}^{k}-n_{L_{g}}^{k}\right) \quad(k=1,2,3) .
$$

Then, eliminating $Y^{3}$ (but not yet imposing the constraint in eq. (3.13)) gives

$$
\frac{5}{3}\left(b_{3}^{\prime 3}-b_{1}^{\prime 3}\right)-\left({b^{\prime}}_{3}^{3}-b_{2}^{\prime 3}\right)=-4+2 \sum_{g=1}^{3}\left(2 n_{Q_{g}}^{3}-n_{u_{g}}^{3}-n_{\ell_{g}}^{3}\right) .
$$

The calculation of the modular weights of the various states on this orbifold was treated in references $[9,22]$ and they are displayed in table 2 . It is easy to satisfy these constraints, including eq. (3.13), for several values of

$$
{b^{\prime}}_{3}^{3}-b_{2}^{\prime 3}=\frac{1}{2}(1,2,3,4)
$$

The cancellation of target-space anomalies in $T^{4}$ requires also that ${b^{\prime}}_{a}^{k}=\delta_{\mathrm{GS}}^{k}$ independently of the gauge group $a$. Hence

$$
b_{3}^{\prime k}=b_{2}^{\prime k}=b_{1}^{\prime k} \quad(k=1,2)
$$

\footnotetext{
${ }^{4}$ These two lattices are actually equivalent, as is shown in table 5.4 of reference Fischer et al. [21]. We are grateful to the referee for drawing our attention to this paper.
} 


\begin{tabular}{|c|c|c|c|c|}
\hline State $X$ & $-n_{X}^{1}$ & $-n_{X}^{2}$ & $-n_{X}^{3}$ & $-\sum_{k} n_{X}^{k}$ \\
\hline$Q, u, \ell$ and $L, h, \bar{h}, d$ & 1 & 0 & 0 & 1 \\
& 0 & 1 & 0 & 1 \\
& 0 & 0 & 1 & 1 \\
& $\frac{1}{2}$ & $\frac{1}{2}$ & 0 & 1 \\
\hline & $\frac{7}{8}$ & $\frac{3}{8}$ & $\frac{3}{4}$ & 2 \\
& $\frac{3}{8}$ & $\frac{7}{8}$ & $\frac{3}{4}$ & 2 \\
& $\frac{3}{4}$ & $\frac{3}{4}$ & $\frac{1}{2}$ & 2 \\
\hline Only $L, h, \bar{h}, d$ & $\frac{15}{8}$ & $\frac{3}{8}$ & $\frac{3}{4}$ & 3 \\
& $\frac{3}{8}$ & $\frac{15}{8}$ & $\frac{3}{4}$ & 3 \\
\hline & $\frac{7}{4}$ & $\frac{3}{4}$ & $\frac{1}{2}$ & 3 \\
& $\frac{3}{4}$ & $\frac{7}{4}$ & $\frac{1}{2}$ & 3 \\
& $\frac{3}{8}$ & $\frac{7}{8}$ & $\frac{7}{4}$ & 3 \\
& $\frac{7}{8}$ & $\frac{3}{8}$ & $\frac{7}{4}$ & 3 \\
\hline & $\frac{23}{8}$ & $\frac{3}{8}$ & $\frac{3}{4}$ & 4 \\
& $\frac{3}{8}$ & $\frac{23}{8}$ & $\frac{3}{4}$ & 4 \\
\hline
\end{tabular}

Table 2. Modular weights of matter representations on the $\mathbb{Z}_{8}$ orbifold.

and

$$
b_{3}^{\prime k}-b_{2}^{\prime k}=0=b_{3}^{\prime k}-b_{1}^{\prime k} \quad(k=1,2) .
$$

Then summing over $k$ gives

$$
\begin{aligned}
b_{3}^{\prime 3}-b_{2}^{\prime 3} & =-6-\sum_{k=1}^{3} Y^{k}+\sum_{g, k=1}^{3}\left(-n_{Q_{g}}^{k}+n_{u_{g}}^{k}\right)>0 \\
\frac{5}{3}\left(b_{3}^{\prime 3}-b_{1}^{\prime 3}\right) & =-18-\sum_{k=1}^{3} Y^{k}+\sum_{g, k=1}^{3}\left(3 n_{Q_{g}}^{k}-n_{u_{g}}^{k}-2 n_{\ell_{g}}^{k}\right)>0 .
\end{aligned}
$$

Now, it is clear from table 2 that the sum $\sum_{k} n_{X}^{k}$ of the modular weights for any particle is an integer, and in fact this is generally true [9]. It then follows from these equations that

$$
\begin{aligned}
b_{3}^{\prime 3}-b_{2}^{\prime 3} & \in \mathbb{Z} \\
\frac{5}{3}\left(b_{3}^{\prime 3}-b_{1}^{\prime 3}\right) & \in \mathbb{Z} \\
\frac{5}{3}\left(b_{3}^{\prime 3}-b_{1}^{\prime 3}\right)-\left({b_{3}^{\prime}}^{3}-b_{2}^{\prime 3}\right) & \in 2 \mathbb{Z} .
\end{aligned}
$$

Evidently the half-integral solutions for $b_{3}^{\prime 3}-b_{2}^{\prime 3}$ in eq. (3.18) are not consistent with the cancellation of modular anomalies. Further, if we now impose the constraint in eq. (3.13), it follows that

$$
b_{3}^{\prime 3}-b_{2}^{\prime 3} \in 2 \mathbb{Z}
$$




\begin{tabular}{|c|c|c|c|}
\hline$-\sum_{k, g=1}^{3} n_{Q_{g}}^{k}$ & $-\sum_{k, g=1}^{3} n_{u_{g}}^{k}$ & $-\sum_{k, g=1}^{3} n_{\ell_{g}}^{k}$ & $-\sum_{k=1}^{3} Y^{k}$ \\
\hline 3 & 6 & 9 & 11 \\
3 & 7 & 8 & 12 \\
3 & 8 & 7 & 13 \\
3 & 9 & 6 & 14 \\
4 & 8 & 9 & 12 \\
4 & 9 & 8 & 13 \\
\hline
\end{tabular}

Table 3. Modular weights satisfying eq. (3.27) when $b_{3}^{\prime 3}-b_{2}^{\prime 3}=2$.

so that only the earlier solutions in which ${b_{3}^{\prime}}^{3}-b_{2}^{\prime 3}=2$ can be consistent with modular anomaly cancellation. Eliminating the $\sum_{k} Y^{k}$ terms from the above equations gives

$$
6+\frac{5}{6}\left(b^{\prime 3}-b_{1}^{\prime 3}\right)-\frac{1}{2}\left({b^{\prime}}_{3}^{3}-b_{2}^{\prime 3}\right)=\sum_{g, k=1}^{3}\left(2 n_{Q_{g}}^{k}-n_{u_{g}}^{k}-n_{\ell_{g}}^{k}\right)
$$

and the modular weights displayed in table 2 allow only the solutions displayed in table 3 when $b_{3}^{\prime 3}-b_{2}^{\prime 3}=2$ (and $\left.b_{3}^{\prime 3}-b_{1}^{\prime 3}=24 / 5\right)$. However, we also need to satisfy eqs. (3.14) and (3.15) with $b_{3}^{\prime 3}-b_{2}^{\prime 3}=2$. The only solutions are displayed in table 4 . The question is whether any of these is achieved by a set of modular weights that also yields one of the solutions given in table 3 . Three of these require $\sum_{g=1}^{3} n_{u_{g}}^{3}=-3$ or $\sum_{g=1}^{3} n_{\ell_{g}}^{3}=-3$. However this can only be achieved for $u_{g}$ if $\left(n_{u_{g}}^{1}, n_{u_{g}}^{2} n_{u_{g}}^{3}\right)=(0,0,1)$ for all three generations. But then $\sum_{k, g=1}^{3} n_{u_{g}}^{k}=-3$ which is not allowed by any of the solutions given in table 3 . The same argument applies to $\ell_{g}$. Similarly, there are two solutions in which $\sum_{g=1}^{3} n_{u_{g}}^{3}=$ $-11 / 4$ or $\sum_{g=1}^{3} n_{\ell_{g}}^{3}=-11 / 4$, and the allowed sets of modular weights satisfying this have $\sum_{k, g=1}^{3} n_{u_{g}}^{k}=-4,-5$, neither of which is allowed by the solutions in table 3 . It follows that the only possibility is the solution with $\sum_{g=1}^{3} n_{u_{g}}^{3}=-5 / 2=\sum_{g=1}^{3} n_{\ell_{g}}^{3}$, which can arise from modular weights with $\sum_{k, g=1}^{3} n_{u_{g}}^{k}=-6,-7=\sum_{k, g=1}^{3} n_{\ell_{g}}^{k}$. Unfortunately none of the solutions in table 3 have both $\sum_{k, g=1}^{3} n_{u_{g}}^{k}=-6,-7$ and $\sum_{k, g=1}^{3} n_{\ell_{g}}^{k}=-6,-7$. The conclusion is that, independently of the moduli-dependence of the threshold corrections, it is impossible to satisfy the unification constraint given in eq. (3.13) consistently with the cancellation of modular anomalies.

In their treatment of this question, instead of imposing eq. (3.13), Ibáñez and Lüst [9] allowed a range of values of

$$
r:=\frac{\frac{5}{3}\left(b_{3}^{\prime 3}-b_{1}^{\prime 3}\right)}{b_{3}^{\prime 3}-b_{2}^{\prime 3}} .
$$

They required that the ratio

$$
\gamma:=\frac{1+r}{\frac{3}{5} r-1}
$$

is in the range

$$
2.7 \leq \gamma \leq 3.7
$$




\begin{tabular}{|c|c|c|c|}
\hline$-\sum_{g=1}^{3} n_{Q_{g}}^{3}$ & $-\sum_{g=1}^{3} n_{u_{g}}^{3}$ & $-\sum_{g=1}^{3} n_{\ell_{g}}^{3}$ & $-Y^{3}$ \\
\hline 0 & 2 & 3 & 6 \\
0 & 3 & 2 & 7 \\
0 & $\frac{9}{4}$ & $\frac{11}{4}$ & $\frac{25}{4}$ \\
0 & $\frac{11}{4}$ & $\frac{9}{4}$ & $\frac{27}{4}$ \\
0 & $\frac{5}{2}$ & $\frac{5}{2}$ & $\frac{13}{2}$ \\
$\frac{1}{2}$ & 3 & 3 & $\frac{13}{2}$ \\
\hline
\end{tabular}

Table 4. Modular weights satisfying eqs. (3.14) and (3.15) when ${b_{3}^{\prime}}^{3}-{b_{2}^{\prime}}^{3}=2$.

corresponding to

$$
3.88 \leq r \leq 5.95
$$

which includes the value $r=4$ corresponding to eq. (3.13). Eqs. (3.23)-(3.25) hold quite generally, and if we choose

$$
b_{3}^{\prime 3}-b_{2}^{\prime 3}=1
$$

then the above range only allows $r=5$ consistently with eq. (3.25), in which case

$$
b_{3}^{\prime 3}-b_{1}^{\prime 3}=3 .
$$

In this case the solutions of eqs. (3.21) and (3.22) are given in table 5, and the solutions of eqs. (3.14) and (3.15) in table 6. The solution given in [9] corresponds to the third line in each table, and it is easily verified that their solution satisfies the modular anomaly cancellation constraints given in eq. (3.19). Thus the $\mathbb{Z}_{8}$-I orbifold does allow gauge coupling constant unification if the weaker constraint given in eq. (3.31) is imposed rather than $r=4$ that is required by eq. (3.13). The outstanding question then is whether this can be achieved with a reasonable value of the Kähler modulus $T_{3}$ of order unity. We shall address this question in section 4 .

\subsection{The $\mathbb{Z}_{3} \times \mathbb{Z}_{6}$ orbifold}

The point group generators $\theta, \omega$ have eigenvalues $e^{2 \pi i v_{k}}$ with

$$
\begin{aligned}
\left(v_{1}, v_{2}, v_{3}\right)_{\theta} & =\frac{1}{3}(0,-1,1) \\
\left(v_{1}, v_{2}, v_{3}\right)_{\omega} & =\frac{1}{6}(1,-1,0)
\end{aligned}
$$

which may be realised using Casimirs on the $\mathrm{SU}(3)^{3}$ lattice. It is convenient to reorder the twists here compared with those used in [13] and table 1 of section 2 , so that now $T_{3}^{2}$ is the $\mathbb{Z}_{3}$-plane, while $T_{1,2}^{2}$ are both $\mathbb{Z}_{6}$-planes. Evidently all three planes $T_{1,2,3}^{2}$ are $\mathcal{N}=2$ planes, so all three Kähler moduli $T_{1,2,3}$ contribute to the threshold corrections. The complex structure moduli $U_{1,2,3}=e^{2 \pi i / 3}$ are all fixed, since none of them is a $\mathbb{Z}_{2}$-plane. In this case 


\begin{tabular}{|c|c|c|c|}
\hline$-\sum_{i, g=1}^{3} n_{Q_{g}}^{i}$ & $-\sum_{i, g=1}^{3} n_{u_{g}}^{i}$ & $-\sum_{i, g=1}^{3} n_{\ell_{g}}^{i}$ & $-\sum_{i=1}^{3} Y^{i}$ \\
\hline 3 & 5 & 9 & 9 \\
3 & 6 & 8 & 10 \\
3 & 7 & 7 & 11 \\
3 & 8 & 6 & 12 \\
3 & 9 & 5 & 13 \\
4 & 7 & 9 & 10 \\
4 & 8 & 8 & 11 \\
4 & 9 & 7 & 12 \\
5 & 9 & 9 & 11 \\
\hline
\end{tabular}

Table 5. Modular weights satisfying eqs. (3.21) and (3.22) when ${b^{\prime}}_{3}^{3}-b_{2}^{\prime 3}=1$.

\begin{tabular}{|c|c|c|c|}
\hline$-\sum_{g=1}^{3} n_{Q_{g}}^{3}$ & $-\sum_{g=1}^{3} n_{u_{g}}^{3}$ & $-\sum_{g=1}^{3} n_{\ell_{g}}^{3}$ & $-Y^{3}$ \\
\hline 0 & 1 & 3 & 4 \\
0 & $\frac{5}{4}$ & $\frac{11}{4}$ & $\frac{17}{4}$ \\
0 & $\frac{3}{2}$ & $\frac{5}{2}$ & $\frac{9}{2}$ \\
0 & $\frac{7}{4}$ & $\frac{9}{4}$ & $\frac{19}{4}$ \\
0 & 2 & 2 & 5 \\
0 & $\frac{9}{4}$ & $\frac{7}{4}$ & $\frac{21}{4}$ \\
0 & $\frac{5}{2}$ & $\frac{3}{2}$ & $\frac{11}{2}$ \\
0 & $\frac{11}{4}$ & $\frac{5}{4}$ \\
0 & 3 & 1 & 6 \\
$\frac{1}{2}$ & 2 & 3 & $\frac{9}{2}$ \\
$\frac{1}{2}$ & $\frac{9}{4}$ & $\frac{11}{4}$ & $\frac{19}{4}$ \\
$\frac{1}{2}$ & $\frac{5}{2}$ & $\frac{5}{2}$ & 5 \\
$\frac{1}{2}$ & $\frac{11}{4}$ & $\frac{9}{4}$ & $\frac{21}{4}$ \\
$\frac{1}{2}$ & 3 & 2 & $\frac{11}{2}$ \\
$\frac{3}{4}$ & $\frac{5}{2}$ & 3 & $\frac{19}{4}$ \\
$\frac{3}{4}$ & $\frac{11}{4}$ & $\frac{11}{4}$ & 5 \\
$\frac{3}{4}$ & 3 & $\frac{5}{2}$ & $\frac{21}{4}$ \\
1 & 3 & 3 & 5 \\
\hline
\end{tabular}

Table 6. Modular weights satisfying eqs. (3.14) and (3.15) when $b_{3}^{\prime}-b_{2}^{\prime}=1$. 
eqs. (3.4) and (3.2) require that

$$
\begin{gathered}
\sum_{k=1}^{3}\left(b_{3}^{\prime k}-b_{2}^{\prime k}\right) \ln \alpha_{k}<0 \\
\sum_{k=1}^{3}\left({b_{3}^{\prime}}^{k}-b_{1}^{\prime k}\right) \ln \alpha_{k}<0 \\
\frac{\sum_{k=1}^{3}\left(b_{3}^{\prime k}-b_{2}^{\prime k}\right) \ln \alpha_{k}}{\sum_{k=1}^{3}\left(b_{3}^{\prime k}-b_{1}^{\prime k}\right) \ln \alpha_{k}}=\frac{5}{12}
\end{gathered}
$$

where

$$
\alpha_{k}:=\left(T_{k}+\bar{T}_{k}\right)\left|\eta\left(p_{k} T_{k}\right) \eta\left(q_{k} T_{k}\right)\right|^{2} .
$$

The quantities $\ln \alpha_{k}$ are dominated the contribution from the Dedekind eta functions whose logarithm is negative in the region of interest, as can be seen in eq. (4.3); this explains the difference between the inequalities in eqs. (3.36) and (3.37) compared with those in eqs. (3.11) and (3.12). If a single modulus, $T_{3}$ say, dominates, these equations reduce to the constraints given previously in eqs. (3.14), (3.15) and (3.13). Further, there are no modular anomalies on the $\mathbb{Z}_{3} \times \mathbb{Z}_{6}$ orbifold. In this case the modular weights $[9,22]$ are given in table 7 , and using these it is clear from eq. (3.14) that $3\left(b_{3}^{\prime 3}-b_{2}^{\prime 3}\right) \in \mathbb{Z}$. In fact, using eq. (3.13), it follows from eq. (3.17) that $3\left({b_{3}^{\prime}}^{3}-{b_{2}^{\prime}}^{3}\right) \in 2 \mathbb{Z}$. Table 8 lists all solutions with $b_{3}^{\prime 3}-b_{2}^{\prime 3}>0$, and shows that the only allowed values are $3\left(b_{3}^{\prime 3}-b_{2}^{\prime 3}\right)=2,4,6,8$; it is easy to see that there are many allowed choices of the modular weights $n_{(h, \bar{h}, d, L) g}^{3}$ that yield the values of $Y^{3}$ that arise.

However, it is probably unrealistic to assume that a single modulus dominates on this orbifold. Instead we assume that

$$
T_{1}=T_{2}=T_{3}:=T
$$

We wish to exploit the considerably reduced modular symmetry that may be associated with the Kähler modulus $T_{3}$ when there are Wilson lines in this $\mathbb{Z}_{3}$ plane. Thus we choose $\alpha_{3} \neq \alpha_{1}=\alpha_{2} ; T_{1,2}^{2}$ are both $\mathbb{Z}_{6}$ planes, so it is perfectly feasible that their modular symmetries are the same (even if there are non-trivial Wilson lines) and that $\alpha_{1}=\alpha_{2}$. Then eq. (3.38) gives

$$
\frac{\sum_{k=1}^{2}\left(b_{3}^{\prime k}-b_{2}^{\prime k}\right) \ln \alpha_{1}+\left(b_{3}^{\prime 3}-b_{2}^{\prime 3}\right) \ln \alpha_{3}}{\sum_{k=1}^{2}\left(b_{3}^{\prime}{ }^{k}-b_{1}^{\prime k}\right) \ln \alpha_{1}+\left(b_{3}^{\prime 3}-b_{2}^{\prime 3}\right) \ln \alpha_{3}}=\frac{5}{12}
$$

where

$$
\alpha_{1}=\alpha_{2}=(T+\bar{T})\left|\eta\left(p_{1} T\right) \eta\left(q_{1} T\right)\right|^{2} .
$$

This is satisfied independently of $\alpha_{1}$ and $\alpha_{3}$ if,

$$
\frac{\sum_{k=1}^{2}\left(b_{3}^{\prime k}-b_{2}^{\prime k}\right)}{\sum_{k=1}^{2}\left(b_{3}^{\prime k}-b_{1}^{\prime k}\right)}=\frac{5}{12}=\frac{b_{3}^{\prime 3}-b_{2}^{\prime 3}}{b_{3}^{\prime 3}-b_{1}^{\prime 3}}
$$




\begin{tabular}{|c|c|c|c|c|}
\hline State $X$ & $-n_{X}^{1}$ & $-n_{X}^{2}$ & $-n_{X}^{3}$ & $-\sum_{i} n_{X}^{i}$ \\
\hline \multirow[t]{2}{*}{$Q, u, \ell$ and $L, h, \bar{h}, d$} & $\frac{5}{6}$ & $-\frac{5}{6}$ & 0 & 0 \\
\hline & $-\frac{5}{6}$ & $\frac{5}{6}$ & 0 & 0 \\
\hline & 1 & 0 & 0 & 1 \\
\hline & 0 & 1 & 0 & 1 \\
\hline & 0 & 0 & 1 & 1 \\
\hline & $\frac{5}{6}$ & $\frac{1}{6}$ & 0 & 1 \\
\hline & $\frac{1}{6}$ & $\frac{5}{6}$ & 0 & 1 \\
\hline & $\frac{1}{3}$ & $\frac{2}{3}$ & 0 & 1 \\
\hline & $\frac{2}{3}$ & $\frac{1}{3}$ & 0 & 1 \\
\hline & $\frac{1}{2}$ & $\frac{1}{2}$ & 0 & 1 \\
\hline & 0 & $\frac{1}{3}$ & $\frac{2}{3}$ & 1 \\
\hline & 0 & $\frac{2}{3}$ & $\frac{1}{3}$ & 1 \\
\hline & $\frac{1}{3}$ & 0 & $\frac{2}{3}$ & 1 \\
\hline & $\frac{2}{3}$ & 0 & $\frac{1}{3}$ & 1 \\
\hline & $\frac{1}{6}$ & $\frac{11}{6}$ & 0 & 2 \\
\hline & $\frac{11}{6}$ & $\frac{1}{6}$ & 0 & 2 \\
\hline & $\frac{5}{6}$ & $\frac{5}{6}$ & $\frac{1}{3}$ & 2 \\
\hline & $\frac{5}{6}$ & $\frac{1}{2}$ & $\frac{2}{3}$ & 2 \\
\hline & $\frac{2}{3}$ & $\frac{2}{3}$ & $\frac{2}{3}$ & 2 \\
\hline & $\frac{1}{2}$ & $\frac{5}{6}$ & $\frac{1}{3}$ & 2 \\
\hline \multirow[t]{22}{*}{ Only $L, h, \bar{h}, d$} & $-\frac{11}{6}$ & $\frac{5}{6}$ & 0 & -1 \\
\hline & $-\frac{11}{6}$ & $\frac{5}{6}$ & 0 & -1 \\
\hline & $-\frac{1}{3}$ & $\frac{1}{3}$ & 0 & 0 \\
\hline & $\frac{1}{3}$ & $\frac{1}{3}$ & 0 & 0 \\
\hline & 0 & $-\frac{1}{3}$ & $\frac{1}{3}$ & 0 \\
\hline & 0 & $\frac{1}{3}$ & $-\frac{1}{3}$ & 0 \\
\hline & $-\frac{1}{3}$ & 0 & $\frac{1}{3}$ & 0 \\
\hline & $\frac{1}{3}$ & 0 & $-\frac{1}{3}$ & 0 \\
\hline & $\frac{1}{3}$ & $\frac{5}{3}$ & 0 & 2 \\
\hline & $\frac{5}{3}$ & $\frac{1}{3}$ & 0 & 2 \\
\hline & 0 & $\frac{1}{3}$ & $\frac{5}{3}$ & 2 \\
\hline & 0 & $\frac{5}{3}$ & $\frac{1}{3}$ & 2 \\
\hline & $\frac{1}{3}$ & 0 & $\frac{5}{3}$ & 2 \\
\hline & $\frac{5}{3}$ & 0 & $\frac{1}{3}$ & 2 \\
\hline & $\frac{11}{6}$ & $\frac{5}{6}$ & $\frac{1}{3}$ & 3 \\
\hline & $\frac{5}{6}$ & $\frac{11}{6}$ & $\frac{1}{3}$ & 3 \\
\hline & $\frac{11}{6}$ & $\frac{1}{2}$ & $\frac{2}{3}$ & 3 \\
\hline & $\frac{1}{2}$ & $\frac{11}{6}$ & $\frac{2}{3}$ & 3 \\
\hline & $\frac{17}{6}$ & $\frac{5}{6}$ & $\frac{1}{3}$ & 4 \\
\hline & $\frac{5}{6}$ & $\frac{17}{6}$ & $\frac{1}{3}$ & 4 \\
\hline & $\frac{11}{6}$ & $\frac{11}{6}$ & $\frac{1}{3}$ & 4 \\
\hline & $\frac{5}{3}$ & $\frac{5}{3}$ & $\frac{2}{3}$ & 4 \\
\hline
\end{tabular}

Table 7. Modular weights of matter representations on the $\mathbb{Z}_{3} \times \mathbb{Z}_{6}$ orbifold. 


\begin{tabular}{|c|c|c|c|c|c|}
\hline$b_{32}^{\prime}{ }^{3}$ & $-\sum_{g} 3 n_{Q_{g}}^{3}$ & $-\sum_{g} 3 n_{u_{g}}^{3}$ & $-\sum_{g} 3 n_{\ell_{g}}^{3}$ & $-3 Y^{3}$ & Table 9 solution \\
\hline$\frac{2}{3}$ & 0 & $(0,1, \ldots, 9)$ & $(9,8, \ldots, 0)$ & $(8,9, \ldots, 17)$ & $=$ II $\supset$ I $\supset$ III $\supset$ IV \\
& 1 & $(2,3, \ldots, 9)$ & $(9,8, \ldots, 2)$ & $(9,10, \ldots, 16)$ & $\supset$ III \\
& 2 & $(4,5, \ldots, 9)$ & $(9,8, \ldots, 4)$ & $(10,11, \ldots, 15)$ & $\supset$ III \\
& 3 & $(6,7,8,9)$ & $(9,8,7,6)$ & $(11,12,13,14)$ & \\
& 4 & $(8,9)$ & $(9,8)$ & $(12,13)$ & \\
\hline$\frac{4}{3}$ & 0 & $(3,4, \ldots, 9)$ & $(9,8, \ldots, 3)$ & $(13,14, \ldots, 19)$ & $=$ II $\supset$ I $\supset$ III $\supset$ IV \\
& 1 & $(5,6, \ldots, 9)$ & $(9,8, \ldots, 5)$ & $(14,15, \ldots, 18)$ & \\
& 2 & $(7,8,9)$ & $(9,8,7)$ & $(15,16,17)$ & \\
& 3 & 9 & 9 & 16 & \\
\hline 2 & 0 & $(6,7,8,9)$ & $(9,8,7,6)$ & $(18,19,20,21)$ & $=$ II $\supset$ I \\
& 1 & $(8,9)$ & $(9,8)$ & $(19,20)$ & \\
\hline$\frac{8}{3}$ & 0 & 9 & 9 & 23 & \\
\hline
\end{tabular}

Table 8. All solutions of eq. (3.17) with ${b_{3}^{\prime}}^{3}-b_{2}^{\prime 3}>0$. The last column indicates which solutions in table 9 wholly or partially realise this solution here.

and this implies that

$$
\frac{\sum_{k=1}^{3}\left(b_{3}^{\prime k}-b_{2}^{\prime k}\right)}{\sum_{k=1}^{3}\left(b_{3}^{\prime k}-b_{1}^{\prime k}\right)}=\frac{5}{12} .
$$

We have just given (in table 8) all solutions of the second equation in equation (3.43). So the question is whether there are solutions of eq. (3.44) that are achievable with the same sets of modular weights as are needed to solve the former.

Summing over $k=1,2,3$, it follows, as in eqs. (3.21) and (3.22), that

$$
\begin{aligned}
\sum_{k=1}^{3}\left(b_{3}^{\prime k}-b_{2}^{\prime}{ }^{k}\right) & =-6-\sum_{k=1}^{3} Y^{k}+\sum_{g, k=1}^{3}\left(-n_{Q_{g}}^{k}+n_{u_{g}}^{k}\right) \\
\frac{5}{3} \sum_{k=1}^{3}\left({b_{3}^{\prime}}^{k}-b_{1}^{\prime}{ }^{k}\right) & =-18-\sum_{k=1}^{3} Y^{k}+\sum_{g, k=1}^{3}\left(3 n_{Q_{g}}^{k}-n_{u_{g}}^{k}-2 n_{\ell_{g}}^{k}\right)
\end{aligned}
$$

where $Y_{k}$ is given in eq. (3.16). As before, we may infer, quite generally, that

$$
\begin{aligned}
\sum_{k=1}^{3}\left(b_{3}^{\prime k}-b_{2}^{\prime k}\right) & \in \mathbb{Z} \\
\frac{5}{3} \sum_{k=1}^{3}\left(b_{3}^{\prime k}-b_{1}^{\prime k}\right) & \in \mathbb{Z} \\
\frac{5}{3} \sum_{k=1}^{3}\left(b_{3}^{\prime k}-b_{1}^{\prime k}\right)-\sum_{k=1}^{3}\left(b_{3}^{\prime k}-b_{2}^{\prime k}\right) & =0 \bmod 2 .
\end{aligned}
$$




\begin{tabular}{|c|c|c|c|c|c|}
\hline Solution & $\sum_{k=1}^{3}\left(b_{3}^{\prime k}-b_{2}^{\prime k}\right)$ & $-\sum_{g, k=1}^{3} n_{Q_{g}}^{k}$ & $-\sum_{g, k=1}^{3} n_{u_{g}}^{k}$ & $-\sum_{g, k=1}^{3} n_{\ell_{g}}^{k}$ & $\sum_{k=1}^{3} Y_{k}$ \\
\hline Ia & 2 & 0 & 5 & 4 & 13 \\
Ib & 2 & 0 & 4 & 5 & 12 \\
IIa & 2 & 0 & 6 & 3 & 14 \\
IIb & 2 & 0 & 3 & 6 & 11 \\
IIIa & 2 & 1 & 6 & 5 & 13 \\
IIIb & 2 & 1 & 5 & 6 & 12 \\
IV & 4 & 0 & 6 & 6 & 16 \\
\hline
\end{tabular}

Table 9. All solutions of eq. (3.50) with $\sum_{k=1}^{3}\left({b_{3}^{\prime}}^{k}-{b_{2}^{\prime}}^{k}\right)>0$.

Imposing eq. (3.44) then gives

$$
3 \sum_{k=1}^{3}\left(b_{3}^{\prime k}-b_{2}^{\prime k}\right)=-12+\sum_{g, k=1}^{3}\left(4 n_{Q_{g}}^{k}-2 n_{u_{g}}^{k}-2 n_{\ell}^{k}\right) .
$$

Thus, in this case

$$
\begin{aligned}
\sum_{k=1}^{3}\left(b_{3}^{\prime k}-b_{2}^{\prime k}\right) & =0 \bmod 2 \\
\sum_{g, k=1}^{3}\left(n_{Q_{g}}^{k}+n_{u_{g}}^{k}+n_{\ell}^{k}\right) & =0 \bmod 3
\end{aligned}
$$

and using the modular weights in table 7 the only solutions are given in table 9 . As before, there are many sets of modular weights that give rise to the values found of $\sum_{k=1}^{3} Y_{k}$.

We first need to know whether any of the solutions displayed in tables 8 and 9 coincide. Consider solution IV of table 9 . From table 7 , we see that $\sum_{g, k=1}^{3} n_{Q_{g}}^{k}=0$ requires that $n_{Q_{g}}^{3}=0$ for all $g$, so this line can only be consistent with some or all of the four lines in table 8 in which $\sum_{g=1}^{3} n_{Q_{g}}^{3}=0$. It also follows from table 7 that $-\sum_{g, k=1}^{3} n_{u_{g}}^{k}=6$ can only be realised with $-\sum_{k=1}^{3} n_{u_{g}}^{k}=2$ for all $g$, and this requires that $-3 n_{u_{g}}^{3}=(0,1,2)$ so that $-\sum_{g=1}^{3} 3 n_{u_{g}}^{3}=(0,1,2, \ldots, 6)$. Similarly for $-\sum_{g=1}^{3} 3 n_{\ell_{g}}^{3}$. Consider the solutions in the first line of table 8 with $b_{3}^{\prime 3}-b_{2}^{\prime 3}=2 / 3$. They have the property that $-\sum_{g} 3 n_{u_{g}}^{3}-\sum_{g} 3 n_{\ell_{g}}^{3}=$ 9. Thus only the four combinations $\left(-\sum_{g} 3 n_{u_{g}}^{3},-\sum_{g} 3 n_{\ell_{g}}^{3}\right)=((3,4,5,6),(6,5,4,3))$ can be realised in this case. The values of $3 Y_{3}$ corresponding to these four solutions are $3 Y_{3}=$ $(10,11,12,13)$. Likewise, only one of the solutions in the line with $b_{3}^{\prime 3}-b_{2}^{\prime 3}=4 / 3$ may be realised, namely the one with $\left(-\sum_{g} 3 n_{u_{g}}^{3},-\sum_{g} 3 n_{\ell_{g}}^{3}\right)=(6,6)$. The solutions with ${b_{3}^{\prime}}^{3}-{b_{2}^{\prime}}^{3}=2$ and $8 / 3$ cannot be realised from solution IV. In summary, using solution IV we can realise only the solutions in table 8 in which

$$
\begin{aligned}
\left(b_{3}^{\prime 3}-b_{2}^{\prime 3},-\sum_{g} 3 n_{Q_{g}}^{3},-\sum_{g} 3 n_{u_{g}}^{3},-\sum_{g} 3 n_{\ell_{g}}^{3}, 3 Y_{3}\right) & =\left(\frac{2}{3}, 0,(3,4,5,6),(6,5,4,3),(10,11,12,13)\right) \\
& =\left(\frac{4}{3}, 0,6,6,16\right) .
\end{aligned}
$$


Also, as above, solutions Ia, Ib, IIa, IIb of table 9 can only be consistent with some or all of the four lines in table 8 in which $\sum_{g=1}^{3} n_{Q_{g}}^{3}=0$. Proceeding as above, for solution Ia, we may infer that for two generations, $g=1,2$ say, $-\sum_{k=1}^{3} n_{u_{g}}^{k}=2$, whereas for the third $-\sum_{k=1}^{3} n_{u_{3}}^{k}=1$. In this last case, $-3 n_{u_{3}}^{3}=(0,1,2,3)$, so that for solution Ia $-\sum_{g=1}^{3} 3 n_{u_{g}}^{3}=(0,1,2, \ldots, 7)$. It follows similarly that $-\sum_{g=1}^{3} 3 n_{\ell_{g}}^{3}=(0,1,2, \ldots, 8)$; for solution $\mathrm{Ib}$, the ranges for $u$ and $\ell$ are interchanged. Thus now we can realise the solutions in table 8 in which

$$
\begin{aligned}
\left(b_{3}^{\prime 3}-b_{2}^{\prime 3},-\sum_{g} 3 n_{Q_{g}}^{3},-\sum_{g} 3 n_{u_{g}}^{3},-\sum_{g} 3 n_{\ell_{g}}^{3}, 3 Y_{3}\right) & =\left(\frac{2}{3}, 0,(1,2, \ldots, 8),(8,7, \ldots, 1),(9,10, \ldots, 16)\right) \\
& =\left(\frac{4}{3}, 0,(5,6,7),(7,6,5), 16\right) \\
& =(2,0,(7,8),(8,7),(19,20)) .
\end{aligned}
$$

Proceeding in this way, we find that the solutions in table 9 generate some of the solutions in table 8 , but not all. Which ones are are displayed in table 10, and for comparison in the last column of table 8 .

Finally, we need to check that the consistent solutions may all be realised by consistent sets of modular weights for $h, \bar{h}, d_{g}, L_{g}$. For example, consider solution Ia in table 9 . The particular choice of the modular weights displayed in table 11 realises the values $(2 / 3,0,4,5,12)$ on the right-hand side of eq. (3.55) and satisfies all of our constraints. The other solutions may be similarly realised.

\section{Estimation of the Kähler modulus needed for unification}

It follows from eq. (3.1) that

$$
\begin{aligned}
-2\left(b_{3}-b_{2}\right) \ln \frac{m_{\text {string }}}{m_{X}} & =\Delta_{3}-\Delta_{2} \\
& =-\sum_{k=1}^{3}\left({b_{3}^{\prime}}^{k}-{b_{2}^{\prime}}^{k}\right) \ln \left[\left(T_{k}+\bar{T}_{k}\right)\left|\eta\left(p_{k} T_{k}\right) \eta\left(q_{k} T_{k}\right)\right|^{2}\right]
\end{aligned}
$$

For all values of interest we may approximate

$$
\ln (\eta(T)) \simeq-\frac{\pi T}{12}
$$

Thus, since $b_{3}-b_{2}=-4$ and $\ln \left(m_{\text {string }} / m_{X}\right) \simeq 2.92$, in general, we seek allowed values of $p_{k}+q_{k}$ for which there are solutions of

$$
23.3 \simeq \sum_{k=1}^{3}\left({b_{3}^{\prime}}^{k}-b_{2}^{\prime k}\right)\left[\frac{\pi\left(p_{k}+q_{k}\right) T_{k}}{6}-\ln \left(2 T_{k}\right)\right]
$$

with $T_{k} \sim 1$. 


\begin{tabular}{|c|c|c|c|c|c|}
\hline Solution & $b_{3}^{\prime k}-{b_{2}^{\prime}}^{3}$ & $-\sum_{g} 3 n_{Q_{g}}^{3}$ & $-\sum_{g} 3 n_{u_{g}}^{3}$ & $-\sum_{g} 3 n_{\ell_{g}}^{3}$ & $-3 Y^{3}$ \\
\hline \multirow{2}{*}{ I } & $\frac{2}{3}$ & 0 & $(1,2, \ldots, 8)$ & $(8,7, \ldots, 1)$ & $(9,10, \ldots, 16)$ \\
& $\frac{4}{3}$ & 0 & $(4,5, \ldots, 8)$ & $(8,7, \ldots, 4)$ & $(14,15, \ldots, 18)$ \\
& 2 & 0 & $(7,8)$ & $(8,7)$ & $(19,20)$ \\
\hline \multirow{2}{*}{ II } & $\frac{2}{3}$ & 0 & $(0,1, \ldots, 9)$ & $(9,8, \ldots, 0)$ & $(8,9, \ldots, 17)$ \\
& $\frac{4}{3}$ & 0 & $(3,4, \ldots, 9)$ & $(9,8, \ldots, 3)$ & $(13,14, \ldots, 19)$ \\
& 2 & 0 & $(6,7,8,9)$ & $(9,8,7,6)$ & $(18,19,20,21)$ \\
\hline III & $\frac{2}{3}$ & 0 & $(2,3, \ldots, 7)$ & $(7,6, \ldots, 2)$ & $(10,11, \ldots, 15)$ \\
& $\frac{4}{3}$ & 0 & $(5,6,7)$ & $(7,6,5)$ & $(15,16,17)$ \\
& $\frac{2}{3}$ & 1 & $(4,5,6,7)$ & $(7,6,5,4)$ & $(11,12,13,14)$ \\
& $\frac{2}{3}$ & 2 & $(6,7)$ & $(7,6)$ & $(12,13)$ \\
\hline \multirow{2}{*}{ IV } & $\frac{2}{3}$ & 0 & $(3,4,5,6)$ & $(6,5,4,3)$ & $(12,13,14,15)$ \\
& $\frac{4}{3}$ & 0 & 6 & 6 & 16 \\
\hline
\end{tabular}

Table 10. Consistent solutions from table 9 .

\subsection{Unification in the $\mathbb{Z}_{8}$-I orbifold}

In the case of the $\mathbb{Z}_{8}$-I orbifold the threshold corrections are determined entirely by the modulus $T_{3}$. As discussed in section 3.1, it is impossible to satisfy the "strong" unification constraint given in eq. (3.13). However if instead, as in [9], we impose the weaker constraint given in eq. (3.31), then there is an allowed solution in which ${b_{3}^{\prime}}^{3}-{b_{2}^{\prime}}^{3}=1$, corresponding to

$$
\frac{b_{3}^{\prime 3}-b_{2}^{\prime 3}}{b_{3}^{\prime 3}-b_{1}^{\prime 3}}=\frac{4}{12} .
$$

In this case, then, we must satisfy

$$
23.3 \simeq \frac{\pi\left(p_{3}+q_{3}\right) T_{3}}{6}-\ln \left(2 T_{3}\right) .
$$

In section 2.2 we showed that, depending on the choice of Wilson lines, the modular symmetry associated with a $\mathbb{Z}_{4}$-plane may be $\Gamma_{0}(2), \Gamma_{0}(4)$ or $\Gamma_{0}(16)$. If we choose Wilson lines $\mathbf{A}_{5}$ and $\mathbf{A}_{6}$ such that $\mathbf{A}_{5}^{t} C \mathbf{A}_{6} \in \mathbb{Z}+\frac{1}{2}$, it is plausible that the threshold corrections involve terms with

$$
p_{3}=16=q_{3}
$$

in which case eq. (4.6) is satisfied when

$$
T_{3} \simeq 1.5
$$

\subsection{Unification in the $\mathbb{Z}_{3} \times \mathbb{Z}_{6}$ orbifold}

As discussed in section 3.2, all three Kähler moduli contribute to the threshold corrections in this case. However, if the modulus $T_{3}$ associated with the $\mathbb{Z}_{3}$-plane dominates, then 


\begin{tabular}{|c|c|c|c|c|}
\hline Particle $X$ & $-n_{X}^{1}$ & $-n_{X}^{2}$ & $-n_{X}^{3}$ & $-\sum_{k} n_{X}^{k}$ \\
\hline$h$ & $\frac{11}{6}$ & $\frac{5}{6}$ & $\frac{1}{3}$ & 3 \\
\hline $\bar{h}$ & $\frac{11}{6}$ & $\frac{1}{2}$ & $\frac{2}{3}$ & 3 \\
\hline$L_{1}$ & 0 & $\frac{1}{3}$ & $\frac{5}{3}$ & 2 \\
\hline$L_{2}$ & $\frac{2}{3}$ & $\frac{2}{3}$ & $\frac{2}{3}$ & 2 \\
\hline$L_{3}$ & $\frac{11}{6}$ & $\frac{1}{2}$ & $\frac{2}{3}$ & 3 \\
\hline$d_{1}$ & $\frac{5}{6}$ & $-\frac{5}{6}$ & 0 & 0 \\
\hline$d_{2}$ & $\frac{5}{6}$ & $-\frac{5}{6}$ & 0 & 0 \\
\hline \multirow[t]{2}{*}{$d_{3}$} & $\frac{5}{6}$ & $-\frac{5}{6}$ & 0 & 0 \\
\hline & $-Y^{1}=\frac{11}{3}$ & $-Y^{2}=\frac{16}{3}$ & $-Y^{3}=4$ & $-\sum_{k} Y^{k}=13$ \\
\hline$Q_{1}$ & $\frac{5}{6}$ & $-\frac{5}{6}$ & 0 & 0 \\
\hline$Q_{2}$ & $\frac{5}{6}$ & $-\frac{5}{6}$ & 0 & 0 \\
\hline$Q_{3}$ & $\frac{5}{6}$ & $-\frac{5}{6}$ & 0 & 0 \\
\hline$u_{1}$ & $\frac{5}{6}$ & $\frac{5}{6}$ & $\frac{1}{3}$ & 2 \\
\hline$u_{2}$ & $\frac{5}{6}$ & $\frac{5}{6}$ & $\frac{1}{3}$ & 2 \\
\hline$u_{3}$ & $\frac{1}{3}$ & 0 & $\frac{2}{3}$ & 1 \\
\hline$\ell_{1}$ & 0 & $\frac{2}{3}$ & $\frac{1}{3}$ & 1 \\
\hline$\ell_{2}$ & 0 & $\frac{1}{3}$ & $\frac{2}{3}$ & 1 \\
\hline \multirow[t]{2}{*}{$\ell_{3}$} & $\frac{2}{3}$ & $\frac{2}{3}$ & $\frac{2}{3}$ & 1 \\
\hline & $\begin{array}{c}b_{32}^{\prime}{ }^{1}=\frac{13}{6} \\
b_{31}^{\prime 1}=-\frac{39}{10}\end{array}$ & $\begin{array}{c}b_{32}^{\prime}{ }^{2}=-\frac{5}{6} \\
b_{31}^{\prime 2}=\frac{71}{10}\end{array}$ & $\begin{array}{l}b_{32}^{\prime}=\frac{2}{3} \\
b_{31}^{\prime}{ }^{3}=\frac{8}{5}\end{array}$ & $\begin{array}{c}\sum_{k} b_{32}^{\prime}{ }^{k}=2 \\
\sum_{k} b_{31}^{\prime k}=\frac{24}{5}\end{array}$ \\
\hline
\end{tabular}

Table 11. Consistent modular weights for solution Ia.

it is certainly possible to satisfy the strong unification constraint. The allowed values of $b_{3}^{\prime 3}-b_{2}^{\prime 3}$, given in table 8 , are

$$
b_{3}^{\prime 3}-b_{2}^{\prime 3}=\frac{1}{3}(2,4,6,8) .
$$

If we choose Wilson lines $\mathbf{A}_{5}$ and $\mathbf{A}_{6}$ in $T_{3}^{2}$ that are not lattice vectors such that $3 \mathbf{A}_{5}^{t} C \mathbf{A}_{6} \in$ $2 \mathbb{Z}$, then the modular group associated with the $\mathbb{Z}_{3}$-plane is $\Gamma_{0}(9)$, whereas if $3 \mathbf{A}_{5}^{t} C \mathbf{A}_{6} \in$ $2 \mathbb{Z}+1$, then it is $\Gamma_{0}(36)$. Table 12 shows how it is possible to satisfy the strong unification constraint with $T_{3} \sim 1$ for all allowed values of $b_{3}^{\prime 3}-b_{2}^{\prime 3}$ by a judicious, but plausible, choice of $p_{3}$ and $q_{3}$.

It is more natural to assume that all three moduli contribute and are of the same size $T_{1} \simeq T_{2} \simeq T_{3}=T$, and we have shown in table 10 that the strong unification condition can still be satisfied, but only for the combinations of $b_{3}^{\prime 3}-b_{2}^{\prime 3}$ and $\sum_{k=1}^{3}\left(b_{3}^{\prime 3}-b_{2}^{\prime k}\right)$ given in the first and third columns of table 13. Wilson lines $\mathbf{A}_{2 k-1}$ and $\mathbf{A}_{2 k}$ in the $\mathbb{Z}_{6}$-planes $T_{k}^{2}(k=1,2)$ are always $E_{8} \times E_{8}$ lattice vectors. If $\mathbf{A}_{2 k-1}^{t} C \mathbf{A}_{2 k} \in 2 \mathbb{Z}$, then the associated 


\begin{tabular}{|c|c|c|c|}
\hline${b_{3}^{\prime}}^{3}-{b_{2}^{\prime}}^{3}$ & $p_{3}$ & $q_{3}$ & $T_{3}$ \\
\hline$\frac{2}{3}$ & 36 & 36 & 0.94 \\
$\frac{4}{3}$ & 36 & 1 & 0.94 \\
2 & 9 & 9 & 1.34 \\
$\frac{8}{3}$ & 9 & 9 & 1.0 \\
\hline
\end{tabular}

Table 12. Choices of $p_{3}$ and $q_{3}$ for the $\mathbb{Z}_{3} \times \mathbb{Z}_{6}$ orbifold that allow unification with $T_{3} \sim 1$.

\begin{tabular}{|c|c|c|c|c|c|c|c|}
\hline$b_{3}^{\prime 3}-b_{2}^{\prime 3}$ & $\sum_{k=1}^{2}\left(b_{3}^{\prime}-b_{2}^{\prime}\right)$ & $\sum_{k=1}^{3}\left(b_{3}^{3}-b_{2}^{\prime k}\right)$ & $p_{3}$ & $q_{3}$ & $p_{1}$ & $q_{1}$ & $T$ \\
\hline$\frac{2}{3}$ & $\frac{4}{3}$ & 2 & 36 & 36 & 1 & 1 & 0.92 \\
$\frac{2}{3}$ & $\frac{10}{3}$ & 4 & 36 & 36 & 1 & 1 & 0.9 \\
$\frac{4}{3}$ & $\frac{2}{3}$ & 2 & 36 & 1 & 1 & 1 & 0.92 \\
$\frac{4}{3}$ & $\frac{8}{3}$ & 4 & 36 & 1 & 1 & 1 & 0.9 \\
2 & 0 & 2 & 9 & 9 & - & - & 1.34 \\
\hline
\end{tabular}

Table 13. Choices of $p_{3}, q_{3}, p_{1}$ and $q_{1}$ for the $\mathbb{Z}_{3} \times \mathbb{Z}_{6}$ orbifold that allow unification with $T \sim 1$.

modular symmetry is the full $\operatorname{PSL}(2, \mathbb{Z})$. Alternatively, if $\mathbf{A}_{2 k-1}^{t} C \mathbf{A}_{2 k} \in 2 \mathbb{Z}+1$, then it is $\Gamma_{0}(4)$. We choose the Wilson lines such that the symmetry is the same in both $\mathbb{Z}_{6}$-planes, and $\alpha_{1}=\alpha_{2}$. Then eq. (4.4) becomes

$$
23.3 \simeq \frac{\pi T}{6}\left[\left({b_{3}^{\prime}}^{3}-b_{2}^{\prime 3}\right)\left(p_{3}+q_{3}\right)+\sum_{k=1}^{2}\left({b_{3}^{\prime}}^{k}-b_{2}^{\prime k}\right)\left(p_{1}+q_{1}\right)\right]-\ln (2 T) \sum_{k=1}^{3}\left(b_{3}^{\prime k}-b_{2}^{\prime k}\right) .
$$

Table 13 shows how it is possible to satisfy the strong unification constraint with $T \sim 1$ for all allowed values of $\left({b_{3}^{\prime}}^{3}-b_{2}^{\prime 3}, \sum_{k=1}^{3}\left({b_{3}^{\prime}}^{k}-b_{2}^{\prime k}\right)\right)$ by a judicious, but plausible, choice of $p_{3}, q_{3}, p_{1}$ and $q_{1}$. (The solution in the last line is the same as the solution in the third line of table 12 because the moduli associated with $T_{1}^{2}$ and $T_{2}^{2}$ do not contribute; in other words, the threshold corrections are dominated by $T_{3}$ in this case.)

\section{Conclusions}

Our main result is that for certain classes of discrete Wilson lines the (Kähler) modular symmetry $\Gamma_{0}(n)$ associated with fixed $\mathbb{Z}_{4^{-}}$and $\mathbb{Z}_{3}$-planes is considerably less $(n=16$ and 36 respectively) than was previously realised. Such planes arise in the $\mathbb{Z}_{8}$ and $\mathbb{Z}_{3} \times \mathbb{Z}_{6}$ orbifolds respectively. Both orbifolds may be realised by Casimirs acting on $T^{4} \oplus T^{2}$ lattices, with $T^{2}$ the $\mathcal{N}=2$ fixed plane. Neither has any fixed $\mathbb{Z}_{2}$-planes, so the only unfixed moduli are Kähler. We also showed that the modular symmetry for $\mathbb{Z}_{6}$ planes may be reduced to $\Gamma_{0}(4)$ for certain classes of discrete Wilson lines.

For the $\mathbb{Z}_{8}$ case, the allowed modular weights of the Standard Model matter content make it impossible to satisfy the "strong" unification constraint for the supersymmetric Standard Model gauge coupling constants given in eq. (3.13) consistently with the cancellation of modular anomalies. It is, however, possible to satisfy a somewhat weaker constraint 
that is compatible with the measured values of the gauge coupling strengths $g_{a}(\mu)$ at low scales $\mu \sim m_{Z}$. In this case the threshold corrections $\Delta_{a}$ depend only on the single modulus $T_{3}$. Using a conjectured form of $\Delta_{a}$ that possesses the reduced modular symmetry $\Gamma_{0}(16)$, we have verified that the gap between the observed unification scale $m_{X} \sim 2 \times 10^{16} \mathrm{GeV}$ and the string scale $m_{\text {string }} \simeq 3.7 \times 10^{17} \mathrm{GeV}$ can now be bridged with a plausible value of $T_{3} \sim 1.5$.

For the $\mathbb{Z}_{3} \times \mathbb{Z}_{6}$ case, the situation is more complicated because the orbifold is realised on a $T_{1}^{2} \oplus T_{2}^{2} \oplus T_{3}^{2}$ lattice and all three tori are $\mathcal{N}=2$ fixed planes. Thus, all three Kähler moduli $T_{1,2,3}$ are unfixed and the threshold corrections depend upon all three; $T_{1}^{2}$ and $T_{2}^{2}$ are fixed $\mathbb{Z}_{6}$-planes, while $T_{3}^{2}$ is a fixed $\mathbb{Z}_{3}$-plane. Since there is no reason why any one of the three moduli should dominate, we assume that all three have the same value $T$. In this case, we find that the allowed modular weights are compatible with the strong unification constraint (for several different values of the allowed anomaly

coefficients $b_{a}^{\prime k}$ ). Further, using the conjectured form of the threshold corrections that possesses the full PSL $(2, \mathbb{Z})$ modular symmetry for $T_{1,2}$ and $\Gamma_{0}(36)$ for $T_{3}$, we showed that the gap between $m_{X}$ and $m_{\text {string }}$ can be bridged in this case too with a plausible value of $T \sim 0.9$. Alternatively, if, notwithstanding our prejudice, a highly anisotropic orbifold is realised with $T_{3}$ the dominant Kähler modulus, then again we find that the allowed modular weights are compatible with strong unification also for several different values of the coefficients $b_{a}^{\prime}{ }^{k}$. Using the conjectured form of the threshold corrections with modular symmetry $\Gamma_{0}(9)$ and/or $\Gamma_{0}(36)$ in this case allows the gap to be bridged with values of $T_{3}$ in the range $0.94-1.34$.

Our results suggest, but do not prove, that the considerably reduced modular symmetry that arises for large classes of discrete Wilson line backgrounds on the $\mathbb{Z}_{8}$ and $\mathbb{Z}_{3} \times \mathbb{Z}_{6}$ orbifolds may well allow the observed unification of the gauge coupling strengths to occur consistently with the scale naturally arising from the heterotic string and with plausible values of the Kähler moduli that determine the overall scale of these orbifolds. Determining if this suggestion is true would require the prior calculation of the threshold corrections in the presence of discrete Wilson lines. Since these Wilson lines mix winding numbers with internal lattice momenta, such a calculation is considerably more complicated than when they are absent.

Open Access. This article is distributed under the terms of the Creative Commons Attribution License (CC-BY 4.0), which permits any use, distribution and reproduction in any medium, provided the original author(s) and source are credited.

\section{References}

[1] J.R. Ellis, S. Kelley and D.V. Nanopoulos, Probing the desert using gauge coupling unification, Phys. Lett. B 260 (1991) 131 [INSPIRE].

[2] U. Amaldi, W. de Boer and H. Furstenau, Comparison of grand unified theories with electroweak and strong coupling constants measured at LEP, Phys. Lett. B 260 (1991) 447 [INSPIRE]. 
[3] V.S. Kaplunovsky, One Loop Threshold Effects in String Unification, Nucl. Phys. B 307 (1988) 145 [Erratum ibid. B 382 (1992) 436] [hep-th/9205068] [INSPIRE].

[4] K.R. Dienes, String theory and the path to unification: A Review of recent developments, Phys. Rept. 287 (1997) 447 [hep-th/9602045] [InSPIRE].

[5] D. Bailin, A. Love, W.A. Sabra and S. Thomas, Duality symmetries of threshold corrections in orbifold models, Phys. Lett. B 320 (1994) 21 [hep-th/9309133] [INSPIRE].

[6] D. Bailin, A. Love, W.A. Sabra and S. Thomas, String loop threshold corrections for $\mathbb{Z}_{N}$ Coxeter orbifolds, Mod. Phys. Lett. A 9 (1994) 67 [hep-th/9310008] [INSPIRE].

[7] M. Spalinski, On the discrete symmetry group of Narian orbifolds, Phys. Lett. B 275 (1992) 47 [INSPIRE].

[8] J. Erler, D. Jungnickel and H.P. Nilles, Space duality and quantized Wilson lines, Phys. Lett. B 276 (1992) 303 [INSPIRE].

[9] L.E. Ibáñez and D. Lüst, Duality anomaly cancellation, minimal string unification and the effective low-energy Lagrangian of $4 D$ strings, Nucl. Phys. B 382 (1992) 305 [hep-th/9202046] [INSPIRE].

[10] J. Erler and M. Spalinski, Modular groups for twisted Narain models, Int. J. Mod. Phys. A 9 (1994) 4407 [hep-th/9208038] [INSPIRE].

[11] D. Bailin, A. Love, W.A. Sabra and S. Thomas, Modular symmetries in $\mathbb{Z}_{N}$ orbifold compactified string theories with Wilson lines, Mod. Phys. Lett. A 9 (1994) 1229 [hep-th/9312122] [INSPIRE].

[12] M.A. Klaput and C. Paleani, The computation of one-loop heterotic string threshold corrections for general orbifold models with discrete Wilson lines, arXiv:1001.1480 [INSPIRE].

[13] A. Love and S. Todd, Modular symmetries of threshold corrections for Abelian orbifolds with discrete Wilson lines, Nucl. Phys. B 481 (1996) 253 [hep-th/9606161] [InSPIRE].

[14] A. Hebecker and M. Trapletti, Gauge unification in highly anisotropic string compactifications, Nucl. Phys. B 713 (2005) 173 [hep-th/0411131] [INSPIRE].

[15] S. Groot Nibbelink and O. Loukas, MSSM-like models on $\mathbb{Z}_{8}$ toroidal orbifolds, JHEP 12 (2013) 044 [arXiv: 1308.5145] [INSPIRE].

[16] H.P. Nilles and P.K.S. Vaudrevange, Geography of Fields in Extra Dimensions: String Theory Lessons for Particle Physics, arXiv:1403.1597 [INSPIRE].

[17] C. Angelantonj, I. Florakis and B. Pioline, A new look at one-loop integrals in string theory, Commun. Num. Theor. Phys. 6 (2012) 159 [arXiv:1110.5318] [INSPIRE].

[18] C. Angelantonj, I. Florakis and B. Pioline, One-Loop BPS amplitudes as BPS-state sums, JHEP 06 (2012) 070 [arXiv:1203.0566] [INSPIRE].

[19] C. Angelantonj, I. Florakis and B. Pioline, Rankin-Selberg methods for closed strings on orbifolds, JHEP 07 (2013) 181 [arXiv:1304.4271] [INSPIRE].

[20] C. Angelantonj, I. Florakis and B. Pioline, Threshold corrections, generalised prepotentials and Eichler integrals, arXiv:1502.00007 [INSPIRE].

[21] M. Fischer, M. Ratz, J. Torrado and P.K.S. Vaudrevange, Classification of symmetric toroidal orbifolds, JHEP 01 (2013) 084 [arXiv:1209.3906] [INSPIRE].

[22] D. Bailin and A. Love, String unification, grand unification and string loop threshold corrections, Phys. Lett. B 292 (1992) 315 [INSPIRE]. 